\section{Indagini Stratigrafiche e Sistemi Informativi Architettonici: il GIS della chiesa di S. Maria in Valle Porclaneta}

\section{Stratigraphical investigations and Architectural Information Systems: the GIS of the church of S. Maria in Valle Porclaneta}

Ilaria Trizio

Università di L'Aquila (Italia)*

\section{Riassunto}

La chiesa di Santa Maria in Valle Porclaneta e i suoi ambienti accessori sono quanto resta di un antico monastero benedettino, edificato nel cuore del territorio abruzzese (Magliano dei Marsi, L'Aquila) sul finire del XII secolo.

Sebbene la letteratura, a partire dalla fine dell'Ottocento, si sia spesso occupata di questo manufatto, il processo di conoscenza storico-critica del complesso si è rivelato difficoltoso a causa dell'assenza di dati archeologici, della scarsità di fonti documentarie pervenute, e, soprattutto a causa del susseguirsi di numerosi interventi di restauro che, succedutisi senza soluzione di continuità per tutto il secolo scorso, ne hanno letteralmente stravolto la compagine originale.

A fronte delle difficoltà incontrate, la sovrapposizione tra i pochi dati documentali e i dati derivati dall'indagine diretta delle strutture, eseguita con I'ausilio di un Sistema Informativo Architettonico ha consentito di ipotizzare una ricostruzione cronologica delle principali fasi di vita del complesso monastico.

Parole chiave: restauro; monasteri benedettini; ricostruzione cronologica.

\begin{abstract}
The church of Santa Maria in Valle Porclaneta and its accessories are what remains of an ancient Benedictine monastery, built in the heart of Abruzzo region (Magliano dei Marsi, L'Aquila) at the end of the XII century.

Even if literature, starting from the end of XIX century, has been often interested in this architectural manufactured, the process of historical-critical knowledge of the complex revealed itself difficult due to the absence of archaeological data, the shortage of the documents and, above all, the numerous interventions of restoration that, followed during all last century, have deeply modified the original compages of it.

Due to the difficulties met, the overlap between the few documental data and the data derived from the direct investigation of the structures, performed with the aid of an Architectural Informative System, made possible to hypothesize a chronological reconstruction of the principal phases of life of the monastic complex.
\end{abstract}

Key words: restauration; Benedictine monasteries; chronological reconstruction.
L'USO DELL'ANALISI STRATIGRAFICA INFORMATIZZATA NELLO STUDIO DELL'ARCHEOLOGIA DEGLI ELEVATI

Il rilievo critico e la stratigrafia degli elevati rappresentano, attualmente, lo stesso strumento di indagine ${ }^{1}$ a servizio della scienza, teorizzata a partire dagli anni settanta come 'archeologia dell'elevato' e riconosciuta oggi come 'archeologia dell'architettura'. Le applicazioni di questa recente disciplina, che possiamo solo ora, a più di trent'anni dalla sua nascita definire come ben strutturata, si sono moltiplicate sia in Italia che all'estero e, le sue modalità operative, seguono regole oramai codificate, che vengono sottoposte periodicamente a revisione ${ }^{2}$ e spesso adattate alle necessità di applicazioni a casi dalle caratteristiche, di volta in volta, uniche. La bibliografia nel settore è vastissima ${ }^{3}$, come anche il dibattito, reso in genere dinamico e interessante dalla natura stessa di questa disciplina che richiede, trasversalmente, la collaborazione multidisciplinare di archeologi, architetti restauratori e storici dell'architettura. Proprio nella sua multidisciplinarietà risiede l'aspetto più interes-

\footnotetext{
- Mentre invece: "Sino a una decina di anni fa si è assistito ad una forte divaricazione tra disegno e rilievo architettonico tradizionali, da un lato, e rilievo archeologico dall'altro: più precisamente l'indagine archeologica - cioè stratigrafica assumeva connotati di estrema specificità scientifica e di metodo nella documentazione del dato materiale, mentre il rilievo architettonico non forniva adeguate risposte soprattutto sul piano della restituzione grafica.» (Mingotto, L., 1999, La cripta della basilica patriarcale di Aquileia. Disegno e rilevamento archeologico dell'architettura storica, in "Archeologia dell'Architettura», n. IV, pp. 159-180) Il gap esistente è stato colmato cercando di far interagire le metodologie di indagine con quelle di restituzione grafica e, soprattutto, cercando di rendere univoco il linguaggio utilizzato da architetti e archeologi.

${ }^{2}$ Periodicamente infatti, vengono pubblicati dei contributi dai quali è possibile trarre un bilancio della situazione nazionale e internazionale della ricerca archeologica in questo settore. Circa dieci anni dopo le prime applicazioni sul campo, Roberto Parenti pone a revisione il metodo sino ad allora utilizzato in Parenti, R., 1992, Fonti materiali e lettura stratigrafica di un centro urbano: risultati di una sperimentazione "non tradizionale», in "Archeologia Medievale», XIX, pp. 7-62; un decennio dopo il precedente, una rilettura critica dell'intera disciplina viene affidata allo scritto, pubblicato postumo, di Isabella Ferrando Cabona: Ferrando Cabona, I., 2002, Guida critica all'archeologia dell'architettura, con premessa di T. Mannoni, in "Archeologia dell'architettura», VII, pp. 9-42. Più di recente è stato invece lo stesso Harris, che nel 1983 con il suo, Principi di stratigrafia archeologica, ha dato vita ad uno strumento operativo fondamentale e di grande efficacia didattica per l'archeologia, ad evidenziare l'importanza della stratigrafia nello studio degli elevati: Harris, E. C., 2003, The stratigraphy of standing structures, con alcune considerazioni in nota di R. Parenti, in «Archeologia dell'architettura", VIII, pp. 9-14.

${ }^{3}$ Non è pensabile, in questa sede, dare delle indicazioni bibliografiche esaurienti ma, tra i testi relativi alle teorie e ai metodi sviluppati si veda: Parenti, R., 1988, Le tecniche di documentazione per una lettura stratigrafica dell'elevato, in Archeologia e restauro dei monumenti, pp. 305-333; Treccani, G. P., 1996, Archeologia stratigrafica e conservazione del costruito: alcuni obiettivi condivisi, in "Archeologia dell'architettura», I, pp. 139-150; Brogiolo, G. P., 1988, Archeologia dell'edilizia storica, Como; Mannoni, T., 1994, Venticinque anni di archeologia globale - 3. Caratteri costruttivi dell'edilizia storica, Genova; Doglioni, F., 1997, Stratigrafia e restauro. Tra conoscenza e conservazione dell'architettura, Lint, Trieste; Pierotti, P. ; Quirós Castillo, J. A., 2000, Archeologia dell'architettura e storia dell'architettura: due discipline a confronto, in Brogiolo, G. P. (a cura di), II Congresso nazionale di archeologia
} medievale (Brescia, 2000), Firenze, All'Insegna del Giglio, pp. 377-380. 
sante dell'archeologia dell'architettura che ha il merito di aver riunificato, attorno allo stesso obiettivo, due figure differenti, ciascuna con un proprio modus operandi: quella dell'archeologo e quella dell'architetto. A prescindere però dalle differenze esistenti tra queste due figure e dai relativi approcci, l'identico obiettivo di conoscenza e di interpretazione dell'edificio è perseguito da entrambe le parti, per cui, tornando alle specifiche metodologiche, tanto il rilievo critico quanto l'analisi stratigrafica degli elevati si basano sullo stesso presupposto, cioè l'osservazione e lo studio analitico delle strutture dell'edilizia storica in modo da ricavare, attraverso l'interpretazione delle stratificazioni, e del rapporto tra le parti edilizie, la successione cronologica degli eventi che ne costituiscono la storia.

Senza ribadire i concetti noti sui quali si fonda l'archeologia dell'architettura, ci basti ricordare che l'analisi stratigrafica degli elevati si applica alle superfici visibili dell'architettura e cerca di dedurre, dalle relazioni esistenti tra le varie unità stratigrafiche riconosciute, ordinate secondo sequenze logico - deduttive, la cronologia relativa del manufatto. Il diagramma stratigrafico, o matrix di Harris, che ne consegue, dunque non è che: «la visualizzazione simbolica del processo di formazione dell'edificio, in cui si compendiano attività costruttive e successive modifiche, compreso il degrado allorchè appare visibile come qualità dei materiali ${ }^{4}$.

Un'applicazione molto particolare dell'analisi stratigrafica, che viene sperimentata con ottimi risultati già da diversi anni, è quella condotta su manufatti già restaurati.

Proprio quest'ultimo tipo di applicazione è quella adottata nello studio condotto sulla chiesa di S. Maria in Valle Porclaneta, in cui, l'approccio stratigrafico su un contesto murario profondamente modificato dai restauri che si sono succeduti senza soluzione di continuità durante tutto il XX secolo, è stato complicato non solo dai risultati stessi del restauro che ha spesso reso incomprensibili le sequenze stratigrafiche, ma anche dalla sua più totale mancanza di documentazione.

\section{I data-base e la gestione informatizzata delle USM}

Sebbene le venga riconosciuto il merito di aver potenziato uno strumento quale l'analisi stratigrafica degli elevati che trae le sue origini dai principi stratigrafici dell'archeologia e dai simbolismi iconici propri del rilievo architettonico, l'archeologia dell'architettura, in quanto scienza recente, è parimenti consapevole dei propri limiti e della perfettibilità degli strumenti operativi che adopera.

\footnotetext{
4 Ferrando Cabona, I., 1998, Problemi di datazione in Archeologia dell'Ar-
} chitettura, in "Archeologia dell'Architettura», n. III, p. 76.
Pertanto, alla presa di coscienza circa l'importanza di riunificare linguaggi, professionalità ed esperienze, ha fatto seguito, purtroppo solo di recente, la necessità di utilizzare, a tale scopo, le nuove tecnologie informatiche.

Tale obiettivo è fondamentale e auspicabile e necessita, ancora una volta, dello sforzo congiunto di archeologi, architetti, informatici. Purtroppo, a tutt'oggi, il sottoutilizzo degli strumenti informatici è un dato di fatto anche se, paradossalmente, si moltiplicano vertiginosamente gli strumenti messi a disposizione, a tale scopo, anche per un'utenza non particolarmente esperta.

L'analisi stratigrafica in particolar modo, sia condotta su uno scavo che su un paramento murario, si presta molto bene ad essere gestita dal calcolatore in tutte le sue fasi: è consigliabile infatti già a partire dal ricorso a fotopiani in scala come base di appoggio del rilievo stratigrafico. Il fotopiano su base fotogrammetrica infatti, a dispetto di qualsiasi rilievo, di per sé è privo di qualsivoglia caratterizzazione interpretativa e, di fatto, rappresenta un primo passo per eludere le problematiche legate alla rappresentazione del manufatto attraverso lo strumento grafico, per sua natura, privo dei caratteri di oggettività necessari ad impostare una buona stratigrafia.

Alla messa a punto del fotopiano segue in genere su di esso il riconoscimento grafico, possibile con qualsiasi software di tipo vettoriale, delle varie USM, che, caratterizzate da un numero identificativo, univoco, possono a loro volta essere facilmente gestite da un qualsiasi database relazionale che abbia, come 'chiave primaria', il numero dell'USM. Quasi tutti i database più comuni, attraverso una progettazione abbastanza semplice, consentono di realizzare interfacce friendly (figure 1 e 2) nelle quali, le relazioni temporali tra le parti sono facilmente leggibili come per le corrispondenti schede cartacee messe a punto dall'Istituto Centrale per il Catalogo e la Documentazione (ICCD).

Tale metodologia, sebbene consenta grande facilità nell'immissione dei dati e la possibilità di immagazzinare una serie cospicua di informazioni alfanumeriche ed immagini ed offra dunque un primo supporto a chi voglia intraprendere l'analisi stratigrafica automatizzata di un edificio, presenta al contempo numerosi limiti dei quali il più evidente deriva dall'impossibilità di collegare topologicamente alla caratterizzazione vettoriale delle USM i dati immessi all'interno del database, e di conseguenza, effettuare delle analisi spaziali.

Alla luce di tali considerazioni è chiaro che la migliore strada percorribile per evitare che le varie fasi che caratterizzano l'analisi stratigrafica viaggino separatamente, ciascuna gestita dal proprio software, è quella di mettere a punto un 


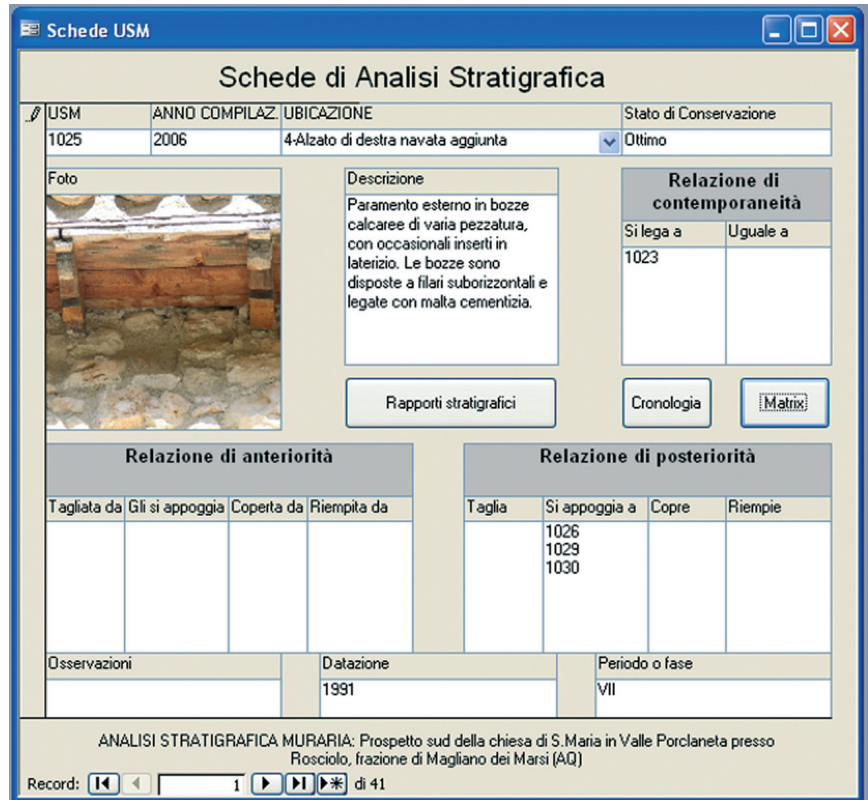

Fig. 1. Maschera del software MS Access creata per la gestione informatizzata delle USM relative all'analisi stratigrafica di un fronte (sud) della chesa di S. Maria in Valle Porclaneta. La maschera illustra le varie información disponibili per una delle USM individuate

progetto GIS, come oramai già assodato dalla maggioranza di coloro che operano nel campo dell'archeologia dell'architettura ed illustrato nei paragrafi che seguono.

\section{GIS a servizio dell'architettura e dell'archeologia}

A circa quaranta anni dalla sua nascita - favorita dalla 'separazione' della scienza cartografica da quella geografica ${ }^{5}$ - quella del GIS (Geographical Information System) può essere considerata una disciplina autonoma a tutti gli effetti, scaturita dall'esigenza, innata nell'uomo, di rappresentare e interpretare al meglio il territorio in cui vive.

Partendo da questa premessa, nella quale è evidente la connotazione spaziale di questa recente 'scienza', è possibile comprenderne la comune definizione, secondo la quale: «è una tecnologia digitale integrata per l'archiviazione, l'analisi, l'organizzazione e la comunicazione di dati spaziali georeferenziati, in pratica tutto ciò che è integrabile con una base cartografica ovvero topologicamente riferita» ${ }^{6}$.

Un GIS, in pratica, è costituito dall'insieme di elementi grafici (vettoriali o raster) collegati topologicamente, tramite attributi comuni, ad un database (cioè un archivio digitale di informazioni). Risulta pertanto evidente che $\mathrm{i}$

\footnotetext{
${ }^{5}$ Salvatori, F., 2002, Geografia e cartografia: da unidea del mondo alla sua gestione, in Spagnoletti, E. (a cura di), 2002, In rete con la comunicazione geografica: atti della quarta conferenza di MondoGis. Roma, 22-24 maggio 2002, Roma, Ed. MondoGIS, pp. 17-19.

${ }^{6}$ Forte, M., 2002, I Sistemi Informativi Geografici in archeologia, Roma, MondoGIS, p. 13.
}

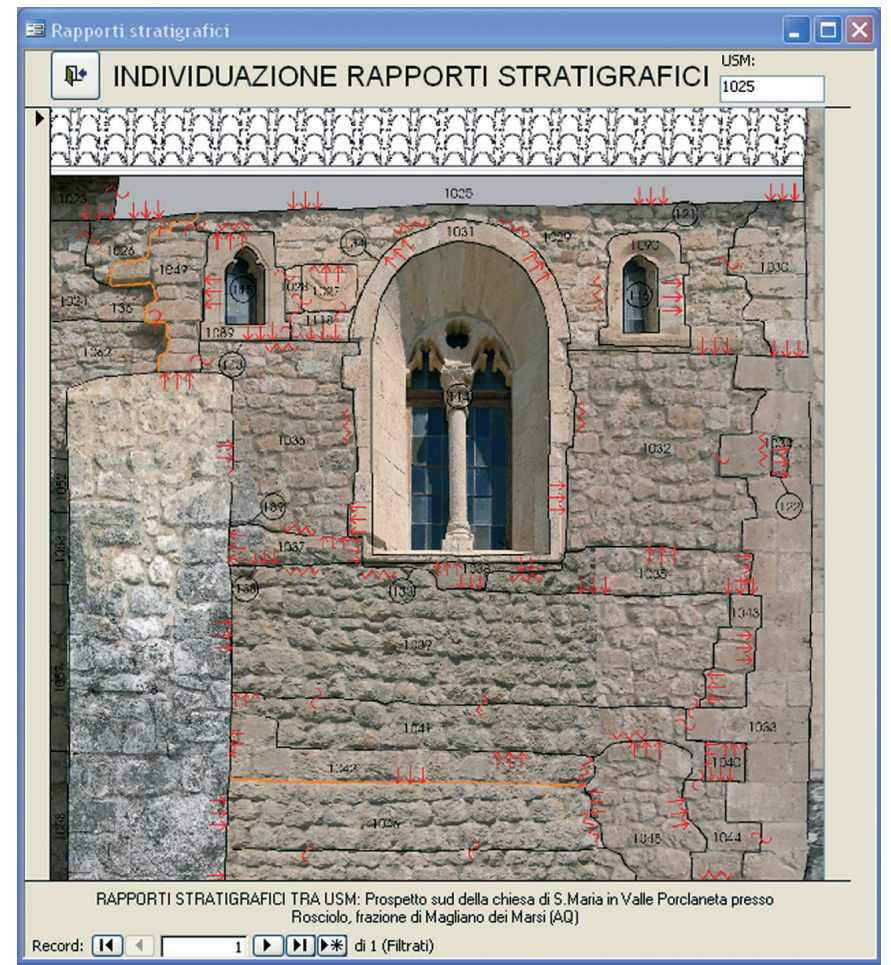

Fig. 2. Maschera del software MS Access collegata alla precedente. La maschera individua graficamente la posizione dell'USM indicata in precedenza ed è attivabile semplicemente cliccando il relativo pulsante 'Rapporti stratigrafici'

dati rappresentano il cuore del GIS, e non solo per il loro contenuto informativo: infatti, la fase determinante nell'ambito della messa a punto di un Sistema Informativo Geografico è quella della progettazione del 'modello dei dati' (dataset) da acquisire, che riveste particolare importanza in quanto in grado di condizionare l'espandibilità del sistema, la sua diffusione e, addirittura, il suo corretto funzionamento.

Un GIS permette dunque di poter gestire contemporaneamente i dati desunti dalla cartografia numerica georeferenziata, quelli descrittivi associati direttamente agli elementi grafici rappresentati e qualsiasi altro tipo di dato anche indirettamente georiferibile ${ }^{7}$.

Sebbene questa disciplina sia nata per soddisfare dei bisogni prettamente legati alla rappresentazione geografica del territorio, un utilizzo particolare della tecnologia GIS, che ha avuto un incremento notevole nell'ultimo decennio, è quello legato al campo della salvaguardia, catalogazione e gestione del patrimonio culturale.

La ragione del successo e della rapida diffusione della tecnologia GIS nel campo dei beni culturali risiede essenzialmente nell'intrinseca connotazione spaziale di scienze come architettura e archeologia, caratteristica riscontrabile

Biallo, G., 2002, Introduzione ai sistemi informativi geografici, Roma, MondoGIS. 
nei siti, nei reperti, negli insediamenti, nei centri storici, negli edifici, nei complessi e nei paesaggi che le costituiscono.

Si è già accennato a come i Sistemi Informativi Territoriali siano complessi di dati alfanumerici e cartografici, informatizzati, e organizzati secondo specifiche architetture, strutturati diversamente a seconda degli scopi che si desidera raggiungere e gestiti da appositi software. Attraverso tali sistemi, la gestione dei dati risulta essere efficiente, dinamica e, soprattutto, costantemente aggiornabile.

Gli archeologi, molto prima di altre figure professionali, hanno compreso le ampie potenzialità offerte dal GIS. Questo interesse si giustifica probabilmente nel metodo che essi abitualmente utilizzano nella gestione dei dati di scavo, analogo a quello necessario ad approntare un qualsiasi Sistema Informativo Geografico. I Sistemi Informativi rispondono molto bene ad esigenze di questo tipo ed inoltre, se ben strutturati, sono in grado di produrre dati che possono essere condivisi da più utenti. I GIS archeologici si suddividono in tre principali tipologie: GIS territoriali o inter-siti (inter-sites), GIS di scavo o intra-sito (intra-site) e GIS del 'territorio costruito'. Questi ultimi sono specifici dell'archeologia dell'architettura, anche se è bene sottolineare che, ovviamente, l'iter metodologico alla base degli uni o degli altri è differente, come anche la scelta dei dataset da immettere e della scala (rispettivamente macro e micro) alla quale operare ${ }^{8}$.

L'utilizzo dei GIS in campo archeologico è ormai consolidato attraverso una pratica che si svolge, seppure con ritmi differenti tra Europa Mediterranea e Stati Uniti, dagli inizi degli anni Ottanta. Nonostante questo, in Italia, a fronte dei numerosi consensi e dell'ampio dibattito metodologico suscitato, il suo impiego è, a tutt'oggi, limitato ad un ristretto numero di gruppi di ricerca, a dispetto invece di quanto accade in altri settori applicativi. La ragione di questa arretratezza sembra risiedere nel fatto che tale tecnologia non sia nata per soddisfare le esigenze della ricerca in campo archeologico, e dunque, lo sforzo effettuato per adattarla a questo nuovo uso richiede tempi maggiori, necessari alla formazione degli operatori e alla modifica dei più comuni software alle specifiche esigenze del settore. Inoltre, è necessario del tempo per vincere la naturale diffidenza, sviluppata da taluni ambiti disciplinari, nei confronti delle tecnologie informatiche, viste ancora spesso solo come un mezzo e non come un vero e proprio settore in evoluzione.

Una novità, sempre in campo archeologico, è rappresentata inoltre dalla possibilità di pubblicare in Internet,

${ }^{8}$ Forte, M., 2002, p. 62 attraverso la creazione di Web-GIS, i risultati delle campagne di scavo. Quello della pubblicazione e diffusione dei dati archeologici è infatti un annoso problema che affligge costantemente i protagonisti della ricerca, favoriti, anche in questo caso, dall'agilità con cui la tecnologia GIS consente loro di gestire i dati accumulati negli anni, nel corso di più campagne successive.

Altra caratteristica rilevante dei Sistemi Informativi Geografici, indispensabile tanto per le indagini archeologiche quanto per quelle architettoniche, è quella di rappresentare un valido supporto operativo necessario alla gestione dei beni culturali. Il GIS, infatti, con la sua produzione di cartografia ed elenchi di dati è uno strumento indispensabile per gli amministratori e i decision-maker in quanto in grado di "prospettare scenari diversi, fornire ipotesi e previsioni, rappresentare conseguenze dirette e indirette di scelte programmatiche, semplicemente attraverso le analisi e l'incrocio dei dati acquisiti»"

Anche in campo architettonico, sebbene recepite con un po' di ritardo rispetto a quello archeologico, non mancano le applicazioni GIS, che, in Italia, vantano numerose esperienze, avviate da varie amministrazioni pubbliche, soprattutto in relazione alla conservazione programmata del patrimonio costruito, alle analisi diagnostiche preventive al progetto di restauro e al monitoraggio del degrado degli edifici storici.

Anche per le applicazioni in campo architettonico, sussiste una ulteriore divisione tra macroscala - a livello territoriale - e microscala - a livello urbano o del singolo manufatto architettonico.

Per quanto riguarda la macroscala, alcune significative esperienze di applicazione al territorio, come ad esempio le analisi condotte sui centri storici italiani, sono state avviate dall'Istituto Centrale per il Catalogo e la Documentazione (ICCD) attraverso il Censimento Nazionale dei Centri Storici, utilizzato come base informativa nazionale unitaria, dalla cui condivisione dei dati sono poi scaturite una serie di interessanti iniziative locali ${ }^{10}$.

Tali iniziative, innestatesi sulla base delle piattaforme GIS nazionali, perseguono gli obiettivi di conoscenza, analisi, tutela e salvaguardia dei caratteri originari, valorizza-

\footnotetext{
${ }^{9}$ Niccolucci, F., 2001, Utilizzo di strumenti free sotware per GIS nel campo dei beni culturali: un esempio di applicazione al patrimonio archeologico, in Gabrielli, G. (a cura di), 2001, Usi e consumi dell'informazione geografica: atti della terza conferenza di MondoGIS. Roma, 23-25 maggio 2001, Roma, Ed. MondoGIS, pp. 201-208.

${ }^{10}$ L'Atlante dei Centri Storici è una banca dati che contiene le schede di censimento di circa 22000 centri storici, suddivise territorialmente, integrate da immagini digitali tratte dalle cartografie IGM attuali e storiche. Per approfondimenti si rimanda al relativo indirizzo web: http://www.iccd.beniculturali.it
} 
Fig. 3. Applicazione della 'cartella clinica dell'edificio' alla chiesa di S. Maria di Collemaggio (L'Aquila). Schermata relativa alla porzione di facciata smontata nel 1915. (Da Bartolomucci, C., 2004b, Santa Maria di Collemaggio. Interpretazione critica e problemi di conservazione, Roma, Palombi, p. 139)

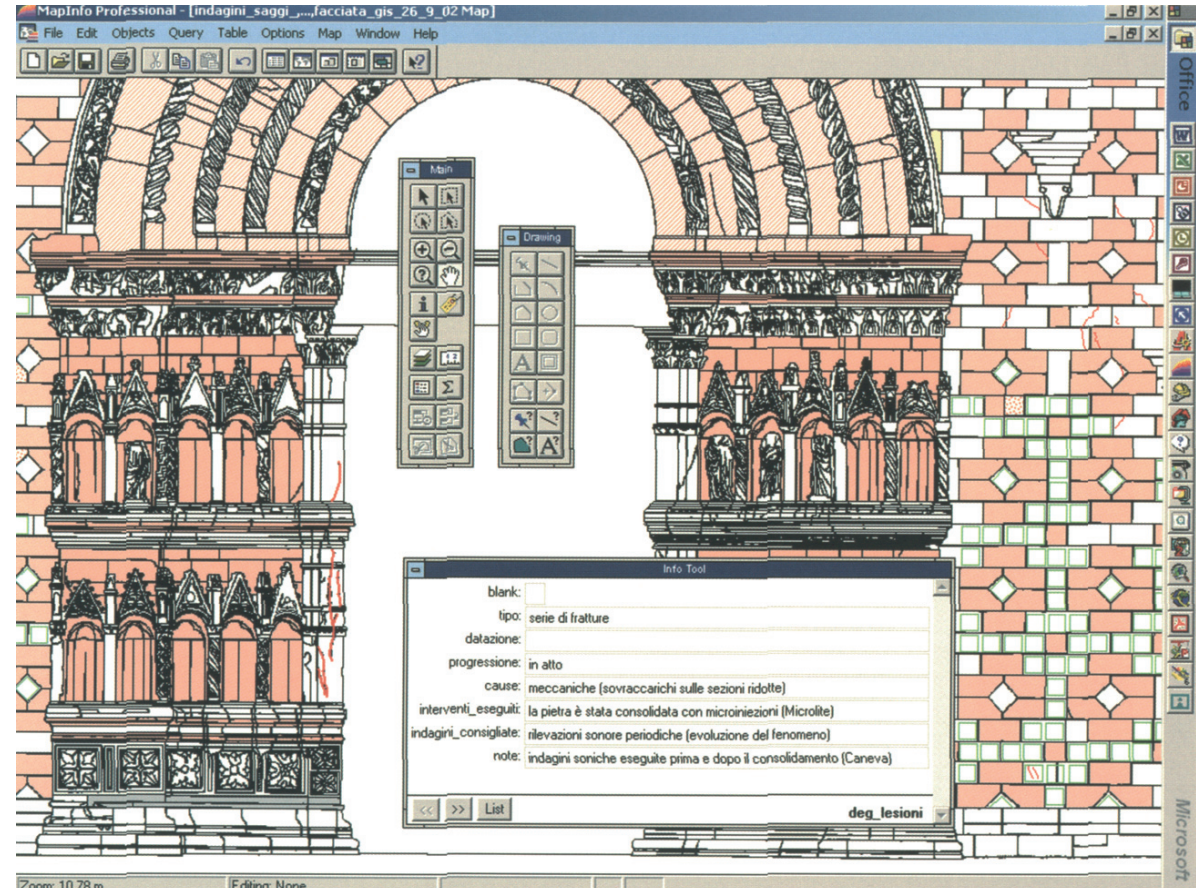

zione e fruizione del patrimonio storico costruito. In quest'ottica si colloca, a titolo d'esempio, come esperienza condotta su macroscala, il sistema Web-GIS per la salvaguardia del patrimonio artistico e architettonico di Venezia. Il progetto, concepito dall'Unesco Venice office come supporto alla pianificazione urbana del centro storico, è stato integrato con il sistema informativo a sostegno degli interventi di restauro, progettato invece dall'associazione scientifica Palazzo Cappello di Venezia. Tale integrazione ha prodotto una cartografia digitale, consultabile dall'utente, attraverso la quale, tramite la selezione di una singola unità edilizia, è possibile reperire informazioni come tipologia, superficie, disposizioni del PRG, dati relativi ai decreti di vincolo degli edifici e risalire, tramite la visualizzazione delle pratiche richieste ai vari enti nel corso degli anni, alla storia conservativa di ciascuna unità edilizia ${ }^{11}$.

L'impiego dei Sistemi Informativi in campo architettonico consente, in buona sostanza, non solo di raccogliere un elevato numero di dati di natura differente, ma anche di integrare e sovrapporre fra loro le informazioni e, grazie soprattutto all'elaborazione e all'analisi statistica, di utilizzare queste ultime, operativamente, nell'ambito del processo decisionale che svolge un ruolo da protagonista nella tutela dei beni.

Le applicazioni più interessanti, nuove e significative di GIS al patrimonio architettonico restano però quelle ${ }^{11}$ Mancuso, A. - Tognon, G., 2003, Un sistema informativo per la salvaguardia del
patrimonio artistico e architettonico di Venezia, in "MondoGIS», n. 37, pp. 31-34. relative alla microscala, nella quale l'analisi viene concentrata su di un unico manufatto architettonico.

Interessanti prospettive in questo senso si sono aperte con la proposta di approntare una 'cartella clinica' del monumento ${ }^{12}$. L'ipotesi della 'cartella clinica' presuppone infatti, per ogni manufatto di accertato interesse storicoartistico, la messa a punto di un GIS che, attraverso la gestione ed elaborazione dei dati raccolti, si ponga come strumento idoneo a strutturare un piano di conservazione e di programmazione degli interventi futuri. Tale strumento è ipotizzato per raccogliere non solo le informazioni tecniche relative allo stato di conservazione di ciascun elemento che compone il manufatto, ma anche le sintesi circa l'analisi storico-critica e le conoscenze tecnico-costruttive relative all'edificio, e che svolga contemporaneamente il ruolo di archivio (attraverso il quale ricostruire e custodire la storia costruttiva e conservativa dell'edificio) e di strumento operativo indispensabile al progetto di restauro (figura 3). Con fine analogo a quello della 'cartella clinica', l'Istituto per le Tecnologie Applicate ai Beni Culturali del $\mathrm{CNR}^{13}$, ha messo a punto un interessante software denominato ARKIS, acronimo di Architecture Recovery Knowledge Information System ${ }^{14}$. L'aspetto

\footnotetext{
${ }^{12}$ Bartolomucci, C., 2004a, Una proposta di 'cartella clinica' per la conservazione programmata, in "Arkos. Scienza e restauro dell'architettura», n.5, pp. 59-65.

${ }^{13}$ Per maggiori informazioni sulle attività dell'Istituto si rimanda al sito internet http://www.itabc.cnr.it.

${ }_{14}$ Per ulteriori informazioni circa la metodologia del progetto, si veda: Salonia, P.; Negri, A., 2002, Conservazione del patrimonio costruito storico: un sistema per
} 
innovativo di questo progetto consiste nell'aver adattato le funzioni caratteristiche dei GIS, alla scala architettonica del manufatto, graficizzato secondo le canoniche rappresentazioni in piante, prospetti e sezioni, e concepito come un'area territoriale specifica compresa all'interno di una cartografia. La struttura metodologica del software si basa sulla combinazione delle normali procedure di rappresentazione CAD (Computer Aided Design) con quelle dei più comuni database relazionali: ne deriva che ogni entità grafica tipica del CAD acquisisce le modalità topologiche e vettoriali dei GIS $^{15}$. Il software, sviluppato con un apposito linguaggio di programmazione, è in grado di analizzare i dati di input (calcolo delle superfici interessate da stesso tipo di degrado, calcolo degli elementi costituiti dallo stesso materiale, calcolo degli elementi architettonici tipologicamente affini per periodo storico, tecnica costruttiva, presenza di patologie, etc.) e di gestire le informazioni attraverso la visualizzazione costante dei dati grafici ed iconografici, opportunamente tematizzati. L'analisi si articola su tre distinti livelli di indagine, corrispondenti a tre differenti scale operative: quella del singolo edificio, quella del settore urbano e quella territoriale; mentre, $i$ dati che è possibile inserire, per ciascun manufatto, riguardano rispettivamente le sfere: anagrafi$\mathrm{ca}$, iconografica, geometrica, strutturale, tecnico-costruttiva e dei materiali, analisi storico-architettonica e degrado. ARKIS, prototipo sperimentato su diversi casi di studio (figura 4), recentemente si è evoluto in ARKIS-NET che è invece un modulo che garantisce la diffusione dei dati (precedentemente generati da ARKIS in forma di GIS), in ambiente Internet ${ }^{16}$.

\section{Applicazioni specifiche: il GIS 'verticale' e I'archeologia dell'architettura}

Dagli ultimi esempi fatti e dalla precedente descrizione della 'cartella clinica', si è potuto intuire come, la logica che permette di approntare un Sistema Informativo Geografico su un manufatto architettonico, sia differente rispetto a quella utilizzata nelle classiche applicazioni territoriali. Infatti, normalmente, un GIS dispone di una base cartografica sulla quale georeferenziare gli elementi, costituita da un piano orizzontale. In questo particolare tipo di applicazioni, invece, che hanno come protagonisti gli edifici, i piani sui quali strutturare i GIS sono, non

lintegrazione e la gestione di dati eterogenei, in Gabrielli, G., pp. 189-194 e Salonia, P.; Negri, A., 2001, ARKIS-NET: un WebGIS per la diffusione della conoscenza sul patrimonio costruito storico, in Spagnoletti, E., pp. 599-603.

${ }^{15}$ Salonia, P.; Negri, A., 2002.

${ }^{16}$ Salonia, P.; Negri, A., 2001. solo quelli, orizzontali, delle piante, ma anche quelli, verticali, rappresentati dalle facciate degli stessi. Proprio grazie a questi ultimi il 'ribaltamento', puramente teorico e di fatto non operativo, del piano, mette in evidenza la principale differenza tra GIS territoriale e del paesaggio, di norma definito 'orizzontale' e GIS architettonico o del territorio costruito, conosciuto invece come 'verticale' ${ }^{17}$. Il GIS del costruito dunque, si presta bene all'analisi dettagliata di micro-contesti (in genere monumentali e architettonici) che rappresentano il campo di azione privilegiato dall'archeologia dell'architettura. L'archeologia dell'architettura infatti, si occupa di tutti i manufatti architettonici che costituiscono, nel loro insieme, i cosiddetti 'paesaggi costruiti'. Tali paesaggi sono caratterizzati da un intrinseco valore di testimonianza, da tutelare e salvaguardare ai fini della trasmissione futura, indipendentemente dalla specifica tipologia, destinazione d'uso o stato di conservazione. Poiché ogni edificio è un'entità geometrica, misurabile e ben definita nello spazio tridimensionale, l'analisi stratigrafica, che parte dal riconoscimento, dal rilievo grafico e dalla catalogazione di ogni singola USM (Unità Stratigrafica Muraria), dimostra, per sua propria natura, vocazione ad essere informatizzata all'interno di un GIS. Tale tecnologia infatti, ben si presta a raccogliere e gestire, in appositi database, le schede descrittive di ciascuna USM e a conservare, grazie alla georeferenziazione, i rapporti topografici esistenti tra USM contigue e tra queste e la superficie d'appartenenza. Come giustamente osservato da alcuni studiosi, però, nell'applicare questa tecnologia a manufatti architettonici, si dovrebbe iniziare a parlare piuttosto che di GIS, di Sistemi Informativi Monumentali o, più propriamente, di Sistemi Informativi Architettonici, visto che l'obiettivo finale è quello di giungere all'analisi monografica del manufatto ${ }^{18}$. Purtroppo però la ricerca in questo settore va ancora notevolmente affinata: il GIS infatti non rappresenta una scienza "ancora sufficientemente evoluta per una conoscenza archeometrica e strutturale del bene alla scala architettonica» ${ }^{19}$, infatti non offre ancora la possibilità di effettuare adeguate elaborazioni e interrogazioni spaziali tridimensionali, indispensabili nell'indagine di complessi volumetrici. Nonostante i problemi tuttora aperti, le esperienze in questo settore si stanno moltiplicando, anche se purtroppo sono

\footnotetext{
${ }^{17}$ "Si definisce un GIS 'verticale’ un sistema informativo spaziale dedicato specificatamente allo studio dell'elevato (territorio costruito) e un GIS 'orizzontale', un sistema orientato prevalentemente all'analisi e rappresentazione del territorio insediato e del paesaggio." (Forte, M., 2002, p. 66).

${ }^{18}$ Forte, M., 2002, pp. 66-67.

${ }^{19}$ Ibid.
} 


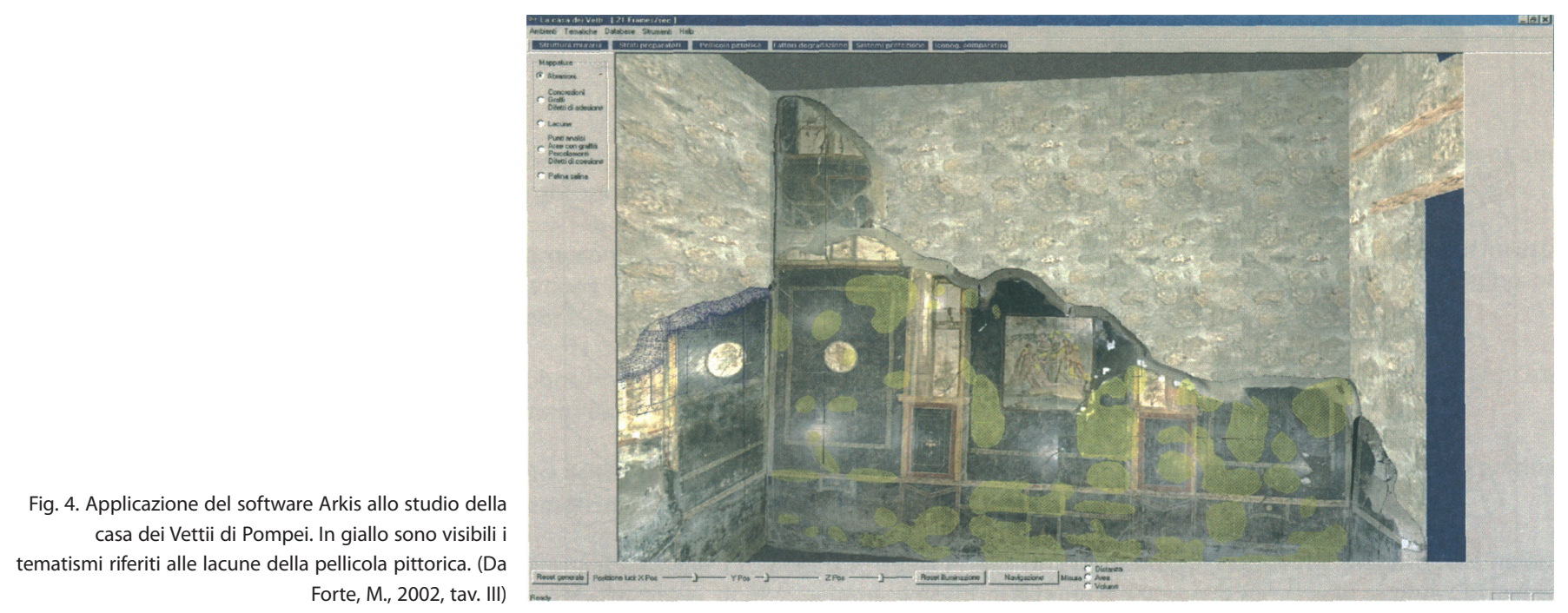

ancora tutte ben lontane dall'essere condotte con omogeneità e sufficienti livelli di standardizzazione ${ }^{20}$.

\section{II futuro della ricerca: GIS tridimensionale e realtà virtuale}

L'uso di modelli tridimensionali all'interno di un GIS rappresenta, in definitiva, un problema tuttora aperto per archeologi, architetti e ricercatori. Le indagini consentite dai più comuni software GIS, attualmente, permettono di condurre numerose analisi spaziali e simulazioni sulla base di modelli virtuali del terreno, come nel caso dei DEM (Digital Elevation Model) o DTM (Digital Terrain Model). Eppure, le crescenti aspettative di archeologi, architetti e operatori dei beni culturali, legate soprattutto alla necessità di 'vedere' e 'percepire' la materia oggetto d'indagine, hanno spinto il GIS ad aprirsi sempre più alla realtà virtuale e al mind-GIS ${ }^{21}$, divenuti strumenti applicativi per la cognizione degli ambienti storici e dei paesaggi archeologici.

Per quanto riguarda la ricostruzione tridimensionale dei paesaggi, a partire da Sistemi Informativi Geografici, quindi da dati georeferenziati, le esperienze effettuate in

\footnotetext{
${ }^{20}$ Cfr. Baracchini, C.; Lanari, P.; Ponticelli, P.; Parenti, R.; Vecchi, A., 2005, SICaR: un sistema per la documentazione georeferenziata in rete, in G. Biscontin, Driussi, G. (a cura di), Sulle pitture murali: riflessioni, conoscenze, interventi. Atti del convegno di studi, Bressanone 12-15 luglio 2005, Marghera-Venezia, Arcadia ricerche, pp. 735-747.

${ }^{21}$ Nella differenza che intercorre tra lo spazio reale del territorio e la sua rappresentazione attraverso la cartografia o il GIS, Maurizio Forte colloca la ricostruzione del paesaggio antico attraverso delle mappe mentali. "Le mappe mentali e cognitive definiscono appunto lo spazio introspettivo (cognitivo) del paesaggio percepito, non un orizzonte meramente geografico-topologico, del visibilevisuale, ma un orizzonte di paesaggi significanti, narrativi, collettivi, impressi nella memoria, antropologizzati." (Forte, M., 2002, p.122) Per ulteriori approfondimenti circa i concetti di mappa mentale, archeologia cognitiva e Mind-GIS si veda GIS e archeologia cognitiva, in Ibid., pp. 119-128.
}

campo nazionale ed internazionale, stando alla letteratura, hanno portato a risultati soddisfacenti, come nelle numerose applicazioni archeologiche di DVR (Desktop Virtual Reality), mentre invece la strada è ancora tutta in salita per le analoghe applicazioni in campo architettonico.

Spesso inoltre, quando si parla di GIS 3D si ingenera confusione tra i programmi che realizzano modelli tridimensionali animati e quelli navigabili interattivamente ${ }^{22}$. La differenza sostanziale tra $\mathrm{i}$ due è che nei primi è possibile raggiungere dei livelli di dettaglio notevoli, non essendo necessario effettuare dei rendering su ogni singola immagine in tempo reale, mentre i secondi, a fronte delle difficoltà scaturite dalla gestione delle immagini che costituiscono l'ambiente virtuale, prevedono la possibilità di interrogare interattivamente il sistema, collegato al dataset d'origine, peraltro dinamicamente aggiornabile. In Italia, una collaborazione attiva tra il VISIT Lab del CINECA e l' ITABC del CNR si occupa di sviluppare applicazioni virtuali con il preciso compito di rispettare la contestualizzazione dell'elemento indagato all'interno del suo paesaggio originale. Tra i vari progetti prodotti da questa collaborazione vi è quello di Desktop Virtual Reality per la ricostruzione virtuale della città di Bologna in età romana, denominato 'Bononia DVRa'. Il progetto si era posto come obiettivo lo studio del paesaggio antico, oramai non più visibile, in relazione a quello attuale. I dati raccolti, come cartografia storica, cartografia attuale, ortofoto, piante e disegni di scavo, database testuali, hanno consentito di costruire un GIS al quale è stato agganciato il sistema DVR che ha permesso di rendere virtualmente

\footnotetext{
${ }^{22}$ In Volo sull'Isola d'Elba: un'applicazione della tecnologia 3D Interactive Map, in
} «MondoGIS», settembre/ottobre 2003, n. 38, pp. 49-52. 


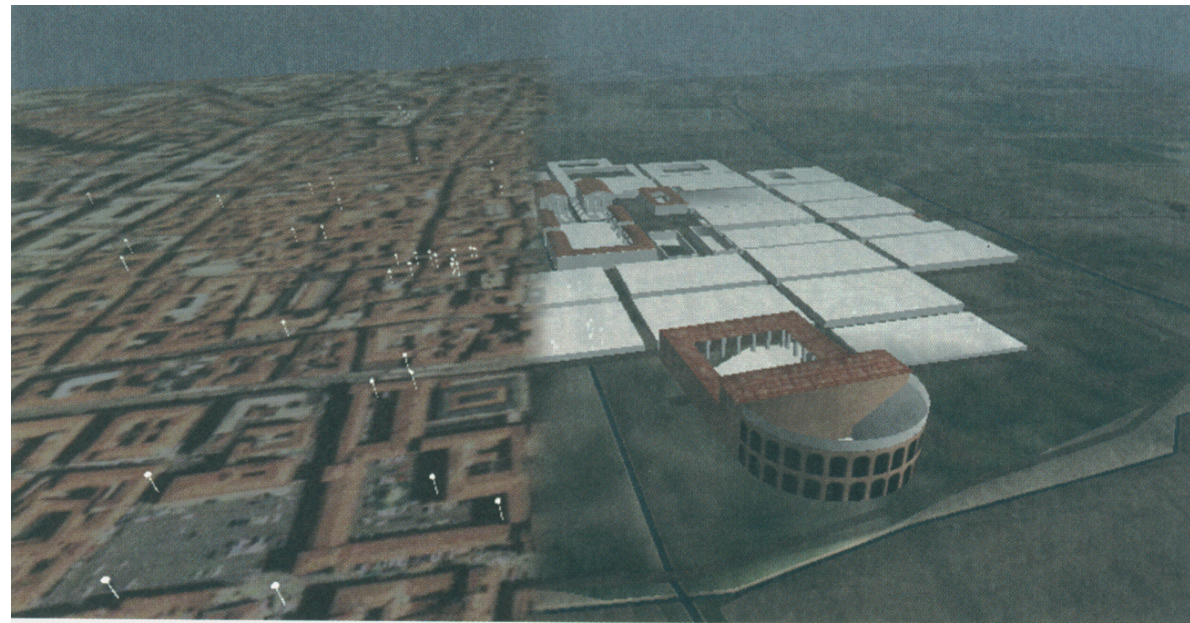

Fig. 5. Applicazione di GIS 3D alla ricostruzione della compagine romana della città di Bologna. (da Forte, M., 2002, tav. XXIV) visibile, e percepibile, una parte del territorio cittadino oramai completamente trasformato (figura 5$)^{23}$.

Anche per quanto riguarda i manufatti architettonici, l'uso di modelli 3D digitali per interpretare la realtà consente di indagare $\mathrm{i}$ rapporti che intercorrono tra le parti componenti l'insieme, in maniera sicuramente più efficace rispetto ai metodi di rappresentazione, per così dire, tradizionali.

D'altra parte, se la componente tridimensionale è importante per la comprensione di un paesaggio naturale, diviene irrinunciabile nei confronti di un'architettura. Di conseguenza, il modello 3D, possibilmente texturizzato, è il più adeguato mezzo di rappresentazione e dunque: "costruendo i modelli digitali come 'sistema conoscitivo' architettonico è possibile estendere facilmente il concetto di GIS (Sistema Informativo Territoriale) 2D a quello di GIS 3D, cosicché ogni modello non è semplicemente un calco, ma un sistema informativo capace di restituire varie proprietà dell'oggetto e analiticità spaziale in modo associativo con la rappresentazione $3 D$ dell'elemento in questione» ${ }^{24}$. E' chiaro allora come il fenomeno legato all'uso dei nuovi sistemi di rappresentazione non si stia diffondendo solo per esaltare le potenzialità dell' Information Tecnology quanto piuttosto in nome di una vera e propria rivoluzione scientifica e culturale "che riporta tutto ciò che era semplicemente valutato qualitativamente ad esserlo quantitativamente, e tutto ciò che era campionato e numerico a divenire visuale $e$ continuo» ${ }^{25}$.

\footnotetext{
${ }^{23}$ Liguori, M. C.; Pescarin, S.; Diamanti, T.; Guidazzoli, A.; Mauri, M. A.; Felicori, M., 2003, Dal GIS alla Realtà Virtuale. Applicazioni per i Beni Culturali e il Decision Making, in «MondoGIS», n. 37, pp. 17-20.

${ }^{24}$ Gaiani, M., 2002, Dal numerico al visuale: strategie di rappresentazione rendering-based, in Biagini, C. (a cura di), Information technology ed automazione del progetto, Firenze, University Press, pp. 30-31.

${ }^{25}$ Ibid.
}

Da quanto visto sinora e dalle previsioni degli addetti ai lavori, il GIS si sta proiettando sempre più verso la multimedialità, e verso applicazioni mirate alla promozione del turismo culturale, per il quale la ricerca è già progredita nel campo della musealizzazzione e della fruizione di parchi archeologici virtuali. Le esperienze di DVR, di semi-immersività all'interno di teatri virtuali o di totale immersività all'interno di cave, rappresentano dunque il futuro di questa tecnologia ${ }^{26} \mathrm{e}$, in definitiva, l'evoluzione culturale del rapporto tra uomo e geografia, secondo cui la mappa digitale non servirà più ad interpretare il territorio ma sarà essa stessa un nuovo territorio, un nuovo tipo di spazio, mentale e geografico ${ }^{27}$.

\section{IL COMPLESSO MONASTICO DI SANTA MARIA IN VALLE PORCLANETA: SINTESI DELLA CRONOLOGIA DOCUMENTATA E FASI COSTRUTTIVE INDIVIDUATE}

Nonostante la controversa ipotesi secondo la quale il monastero di Santa Maria in Valle Porclaneta, edificato nella Marsica, nel cuore dell'Abruzzo aquilano, sia stato

\footnotetext{
${ }^{26}$ Le applicazioni di realtà virtuale si distinguono infatti in DVR, in esperienze collettive semi-immersive all'interno di teatri virtuali e in esperienze totalmente immersive all'interno di cave. Delle prime, che consentono la visualizzazione tridimensionale in tempo reale, si è già diffusamente parlato. Le seconde, invece, si realizzano in ambienti dotati di un ampio schermo semicircolare, di poltrone opportunamente orientate e di un sistema sonoro digitale tridimensionale. In questi ambienti, i teatri virtuali appunto, vengono realizzate le proiezioni delle scene che, grazie alla particolare conformazione dell'ambiente, amplificano la percezione tridimensionale delle stesse. La cave infine, è un ambiente particolare, costituito da una stanza quadrangolare in cui ogni parete è uno schermo proiettivo. L'utente all'interno di questo spazio, durante la proiezione, non ha più la possibilità di percepire la differenza tra ambiente virtuale e reale.

27 "In questo nuovo contesto descrittivo-connettivo, in cui lo spazio telematicovirtuale ospiterà innumerevoli e indefiniti clusters e modelli di dati, potrà forse dirimersi il rapporto dialettico fra «mappa» e territorio: la mappa digitale non sarà il territorio ma un nuovo territorio, uno spazio cognitivo condiviso, mentale e geografico (MindGIS).» (Forte, M., 2002, p. 181).
} 
eretto tra l'VIII e il IX secolo su un sito precedentemente occupato da un santuario romano-italico ${ }^{28}$, la sintetica ricostruzione cronologica del complesso si fa partire dagli eventi successivi all'acquisizione dello stesso da parte dei monaci cassinati, avvenuta, secondo le fonti, tra il 1064 e il 107729. L'arrivo dei benedettini nell'area, negli ultimi decenni dell'XI secolo, coincide con un generale rinnovamento del complesso originario ${ }^{30}$, diretto dall'architetto Niccolò $^{31}$. A questo periodo probabilmente risale l'aula dell'edificio ecclesiastico, suddivisa in tre navate dalla doppia fila di pilastri i cui capitelli, accanto ad una prima rozza lavorazione della pietra a motivi vegetali, zoomorfi ed antropomorfi, recano scolpita o a volte solo abbozzata la 'cornice classica benedettina', secondo la definizione data dalla letteratura ${ }^{32}$, parimenti riscontrabile su molte chiese abruzzesi, benedettine, coeve. Nel 1137 il monastero è ancora di proprietà cassinese, secondo quanto attestato da un documento di Lotario III $^{33}$ che ne conferma le immunità, i privilegi e le rendite. Sempre nel XII secolo, in un periodo di evidente vitalità per il cenobio, la chiesa viene arricchita con importanti arredi liturgici, in particolare ciborio ed ambone, probabilmente completati entrambe nell'anno 1150 , secondo quanto si evince dall'epigrafe incisa sulla balaustra del secondo. Tra la fine del 1160 e gli inizi del 1161, il Chronicon di Fossae Novae ${ }^{34}$ riporta che nella marsica si verificano vari episodi sismici ai quali la critica, in maniera piuttosto unanime, suole attribuire il rinnovamento di molti edifici ecclesiastici nell'area, denunciato dalla presenza di elementi lapidei romanici reimpiegati. A questo evento probabilmente risale il diverso impiego dei plutei, analoghi a quelli presenti sul lato sinistro del recinto presbiteriale, utilizzati per edificare l'altare (rimosso poi nel secolo scorso) posto sotto l'ambone. Nel 1191, si apprende, da un decreto dell'Imperatore

${ }_{28}$ Grossi, G., 2004, Marsica sacra. Chiese, Celle e Monasteri (IV-XII secolo), Avezzano (L'Aquila), p. 93.

${ }^{29}$ In questo intervallo di tempo infatti la critica inserisce la donazione di Berardo, conte dei Marsi, ai benedettini di Montecassino, anche se, da parte delle fonti cassinesi la prima ed unica menzione del cenobio risale al 1077 (cfr. Chronicon Monasterium Casinensis, Lib.III, cap. LXI, in Hoffmann, H. (a cura di), 1980, Die Chronik von Montecassino, MGH, Scriptores, XXXIV, Hannoverae, Ed. Hoffmann, p. 441).

${ }^{30}$ D'Antonio, M., 2003, Abbazie benedettine in Abruzzo, Pescara, Carsa, p.72.

${ }^{31}$ Tale intervento è attestato dall'iscrizione sul monolite d'ingresso (cfr. De Spirito, G., 2002, Su talune iscrizioni medievali di S.Maria in Valle Porclaneta, in La terra dei Marsi. Cristianesimo, cultura, istituzioni, p. 446).

${ }^{32}$ Gavini, I. C., 1927, Storia dell'architettura in Abruzzo, Milano-Roma, Bestetti e Tumminelli, s.a. [ma 1927], vol. I, p. 50.

${ }^{33}$ Diploma di Lotario III del 1137, in Fabiani, L., La terra di S.Benedetto. Studio storico-giuridico sull'Abbazia di Montecassino dall'VIII al XIII secolo, Miscellanea Cassinese 33-34-42, Badia di Montecassino, 1968-80, vol. II, 427.

${ }^{34}$ Regesto riportato da Ricci, C., 1915, in AA.VV., I danni all'arte nei paesi battuti dal terremoto del 13 gennaio 1915, Roma, Calzone, p. 2.
Enrico VI, che il monastero è ancora di proprietà Cassine$\mathrm{se}^{35}$. Nel XIII secolo, maestranze operanti nell'area marsicana realizzano le quattro colonne disposte sopra il recinto presbiteriale e l'articolato fronte absidale esterno nonchè forse, anche il campanile su più piani, con la classica successione ai differenti livelli di monofore, bifore e trifore, già in stato di rudere all'epoca del sisma del 1915 e completamente rimosso durante gli anni trenta del secolo scorso $^{36}$. Al 1268, anno della sconfitta di Corradino di Svevia da parte di Carlo d'Angiò, gli storici assegnano la distruzione del monastero da parte delle truppe tedesche ${ }^{37}$. L'evento, mai confermato né tantomeno smentito dalla critica successiva, potrebbe giustificare la mancanza, a partire da quella data, di documenti che attestino la presenza del cenobio all'interno dei possedimenti cassinesi nell'area fucense. Il monastero, o quel che ne resta, con l'avvento degli angioini passa sotto la dominazione regia, inaugurando un periodo in cui si succederanno varie commende $^{38}$. Successivamente infatti, la prepositura della chiesa passa ai membri della famiglia Orsini ${ }^{39} \mathrm{e}$, nel 1352, Papa Innocenzo VI, con una bolla del 1353 conferisce la chiesa al Cardinale Rinaldo Orsini, sancendone la giurisdizione al vescovo dei Marsi ${ }^{40}$.

A partire da questa data e per tutto il corso del XV secolo, come prevedibile a causa del suo consistente patrimonio, la chiesa inizia a divenire oggetto di contesa fra papato e famiglie reali. Nel 1420 la prepositura viene assegnata, da Giovanna II D'Angiò, che ne ha ancora pieno diritto, alla famiglia dei Colonna ${ }^{41}$. A questo periodo probabilmente risalgono i lavori effettuati sulla chiesa, lungo il fronte sud, tra il 1424 ed il 1430, dalla contessa

\footnotetext{
35 Dragonetti, G., 1765, Difesa del Regio Padronato di S.Maria della Valle Porcanete, Napoli, p. 4.

${ }^{36}$ Nonostante la critica sia stata sempre compatta nell'asserire che il campanile romanico posto in facciata sia andato definitivamente in rovina a causa del sisma del 1915, dalla consultazione di alcuni schizzi autografi dell'architetto Francesco Caracciolo, datati 1830 e conservati presso il fondo Lanciani della Biblioteca di Archeologia e Storia dell'Arte di Palazzo Venezia a Roma, è emerso come, addirittura un secolo prima della sua completa rimozione, la torre campanaria fosse già nello stesso stato di degrado desumibile dalle fotografie degli anni trenta.

${ }^{37}$ Ipotesi accennata, prima dal Phoebonius (in Phoebonius, M., 1678, Historiae Marsorum, vol.III, Napoli, testo latino e traduzione a cura dell'I.N.E.S.A., Roma, De Cristofaro, 1991, p. 175) e poi da Dragonetti (in Dragonetti, G., 1765, p. 5).

${ }^{38}$ Phoebonius, M., 1678 , p. 175.

${ }^{39}$ L'evento è dimostrato dalla donazione, eseguita nel 1334, da parte del prevosto della chiesa, Cardinale Napoleone Orsini, della preziosa croce processionale in argento, di proprietà della propria famiglia (in Nardecchia, P., 2004, Note d'arte abruzzese tra la Marsica e il Carseolano, Carsoli (L'Aquila), Ass. Culturale Lumen, pp. 94-95).

${ }^{40}$ Phoebonius, M., 1678, p. 175.

${ }^{41}$ Bindi, V., 1889, Monumenti storici artistici degli Abruzzi. Studi di V.B. con
} prefazione di F. Gregorovius, Napoli, Giannini \& F., vol. II, p. 901. 
Jacovella di Celano, come è testimoniato dalla presenza delle due monofore a lato della bifora tardogotica, per alcuni studiosi ottenute con il reimpiego di elementi prelevati dalle rovine della vicina chiesa di Santa Maria della Vittoria ${ }^{42}$, e lo stemma nobiliare dei conti Berardi di Celano, inserito a sinistra della bifora. Probabilmente, a questo stesso periodo corrisponde l'intera sistemazione del braccio longitudinale a sud dell'edificio, nel quale vengono inserite non solo le suddette aperture, ma anche l'accesso architravato con le due finestrelle votive ai lati, inserito nell'ispessimento della muratura lungo lo stesso fronte. Con l'avvento degli aragonesi, nel 1441, il diritto di prepositura passa al Re di Napoli Ferdinando I ${ }^{43}$ e, sino alla fine del XV secolo la prepositura della chiesa continua ad essere affidata a uomini di regia dipendenza ${ }^{44}$. A partire dagli inizi del XVI secolo, alla contesa fra l'autorità regia e pontificia si aggiunge una lotta intestina fra le abbazie di Farfa e Montecassino, con la diocesi dei Marsi, contro le famiglie Orsini e Colonna, guerra che verrà combattuta a colpi di bolle emesse ora dagli uni ora dagli altrii ${ }^{45}$. Solo nel 1836 la questione potrà dirsi finalmente chiusa, con l'emissione della bolla di Papa Gregorio XVI, nella quale si attribuisce alla Diocesi dei Marsi il beneficio della chiesa ed il relativo diritto di nomina ${ }^{46}$. Nonostante il grande interesse economico che ha sempre suscitato, il XIX secolo si chiude, per la nostra fabbrica, in un clima di totale abbandono $^{47}$ e generale decadenza, specialmente dopo l'entrata in vigore delle leggi di alienazione dei beni ecclesiastici, come viene denunciato più volte agli inizi del secolo successivo dall'ispettore della Soprintendenza Antonio De Nino che cerca di interessare l'opinione pubblica allo stato di degrado dell'edificio ${ }^{48}$. I due terremoti che si susseguono, quello del 1904, che non provoca danni

\footnotetext{
${ }^{42}$ Ipotesi avanzata, con non pochi dubbi, da Mancini (in Mancini, R., 2003, Viaggiare negli Abruzzi, vol. I, L’Aquila, Textus, pp. 295).

${ }^{43}$ Come attesta l'iscrizione su un affresco interno alla chiesa, nella quale vi è testimoniata la visita di un messo reale in data 1444 (Santa Maria in Valle Porclaneta di Rosciolo, 2002, a cura della Soprintendenza per i Beni Architettonici e per il Pesaggio, per il Patrimonio Storico, Artistico e Demoetnoantropologico dell'Abruzzo - L'Aquila, L'Aquila, p. 79).

${ }^{44}$ Dragonetti, G., 1765, p. 19.

${ }^{45}$ Angeloni, V., 2000, Santa Maria in Valle Porclaneta (Sec.XI). Storia, Arte, Leggende e Tradizioni, Avezzano (L’Aquila), Sogeas, pp. 36-37.

${ }^{46}$ Archivio Diocesano dei Marsi (ADM), fondo C/78/71528.

47 Infatti, da una relazione del marzo 1889, compilata dal Genio Civile di Avezzano, corredata da numerosi schizzi e rilievi, si evince la drammatica situazione in cui versa la chiesa con, in particolare, le prime due arcate sulla sinistra tamponate, probabilmente per alleggerire i pilastri, già vistosamente lesionati a causa del peso della muratura soprastante (Archivio Centrale dello Stato di Roma, Ministero della Pubblica Istruzione, fondo Antichità e Belle Arti, II versamento, II serie).

${ }^{48}$ De Nino, A., 1904, Sommario dei monumenti e degli oggetti d'arte descritti da
} Antonio De Nino, Vasto (Chieti), Anelli, p. 66. evidenti, e quello del 1915, che produce notevoli dissesti ${ }^{49}$, specie sulla calotta absidale, in alcuni pilastri e negli archi a tutto sesto, contribuiscono a peggiorare lo stato delle $\operatorname{cose}^{50}$. Un primo importante ciclo di restauri nel quale si cerca principalmente di intervenire sulla statica dell'edifi$\mathrm{cio}^{51}$, viene compiuto negli anni $1930-31^{52}$, proseguito poi nel 1942 e interrotto dopo solo un anno a causa degli eventi bellicis3 ${ }^{3}$. Numerosi altri interventi, sulla struttura dell'edificio e sugli arredi liturgici, nonché sugli affreschi, continuano a susseguirsi per tutto il secolo ${ }^{54}$; fra questi, di maggior rilievo sono l'intervento di sistemazione esterna operato dalla Soprintendenza ai Monumenti nel $1967^{55}$ e l'adeguamento antisismico compiuto tra il 1990 e il $1991^{56}$. Al restauro strutturale dell'edificio infine, ha fatto seguito, sempre nel 1991, quello alle opere d'arte, con la stessa direzione dei lavori precedente ${ }^{57}$.

${ }^{49}$ Castenetto, S. - Galadini, F. (a cura di), 1999, 13 gennaio 1915. Il terremoto nella Marsica, Roma, pp. 280-282.

${ }^{50}$ Mastroddi, M., 1999, I monumenti danneggiati nella Marsica, in Csastenetto Galadini, 1999, pp. 373-403.

${ }^{51}$ Viene demolito e ricostruito il braccio sinistro della chiesa con il fronte nord; vengono eseguiti i lavori di sbancamento del terreno che si addossava al fronte nord predetto, vengono rimossi i ruderi del campanile romanico in facciata e realizzato lo sperone murario addossato al nartece.

52 Archivio Storico della Soprintendenza ai Beni Ambientali, Architettonici, Artistici e Storici dell'Aquila, cartella E.E.M. 907.

${ }^{53}$ I principali lavori, eseguiti dal Genio Civile dell'Aquila in collaborazione con la Soprintendenza prima dell'interruzione dovuta al secondo conflitto mondiale, provvedono allo smontaggio delle coperture, al consolidamento di tutte le murature con una serie di riprese a 'cuci e scuci', alla realizzazione di cordoli in cemento armato alla sommità dei muri periferici e di spina, allo smontaggio parziale e ricomposizione di quattro pilastri che presentano sintomi da schiacciamento, alle colature di legante liquido in alcune lesioni dei paramenti (Chierici, U., 1945, Relazione sull'attività dell'Ufficio nel quadriennio 1942-45, Aquila, pp. 13-14).

${ }^{54} \mathrm{La}$ seconda parte dell'intervento interrotto a causa della guerra ha riguardato principalmente il restauro degli arredi liturgici (Archivio Storico della Soprintendenza ai Beni Ambientali, Architettonici, Artistici e Storici dell'Aquila, cartella E.E.M. 907)

${ }_{55}$ L'intervento, progettato e diretto dalla Soprintendenza dell'Aquila, riguarda principalmente la sistemazione esterna dell'edificio (cfr. Moretti, M., 1972, Restauri d'Abruzzo (1966-1972), Roma, De Luca, pp. 254-257).

${ }^{56}$ Nell'ambito di questo intervento, progettato e diretto dalla Soprintendenza dell'Aquila, si è provveduto al preconsolidamento di tutte le strutture in elevazione tramite perforazioni armate; perforazioni di consolidamento ai pilastri; smontaggio e rimontaggio del pavimento in pietrame irregolare; spicconatura e rifacimento degli intonaci interni; consolidamento e restauro della cortina in pietra squadrata e di quella in pietra irregolare all'esterno e perforazioni dello sperone lungo il fronte sud; rimontaggio e consolidamento dell'architrave in pietra; realizzazione di un'intercapedine lungo il lato nord dell'edificio e relative perforazioni armate lungo tutto il suo sviluppo; restauro della cortina in pietra squadrata degli archi a tutto sesto interni; perforazioni di fondazione ed inserimento di una catena filettata lungo i quattro lati dell'aula; scavo di sbancamento nel pavimento della chiesa, ad esclusione del corpo aggiunto, per consentire l'inserimento delle travi armate di fondazione; perforazioni lungo i muri perimetrali per consentire l'aggancio dei ferri delle travi di fondazione.

${ }^{57}$ Notizie relative a quest' ultimo intervento sono tratte da Sonnino, E., S.Maria in Valle Porclaneta. Note sulla storia conservativa e le caratteristiche tecnicostilistiche di alcune opere attraverso il loro restauro, in La terra dei Marsi. Cristianesimo, cultura, istituzioni, 2002, pp. 427-436. 


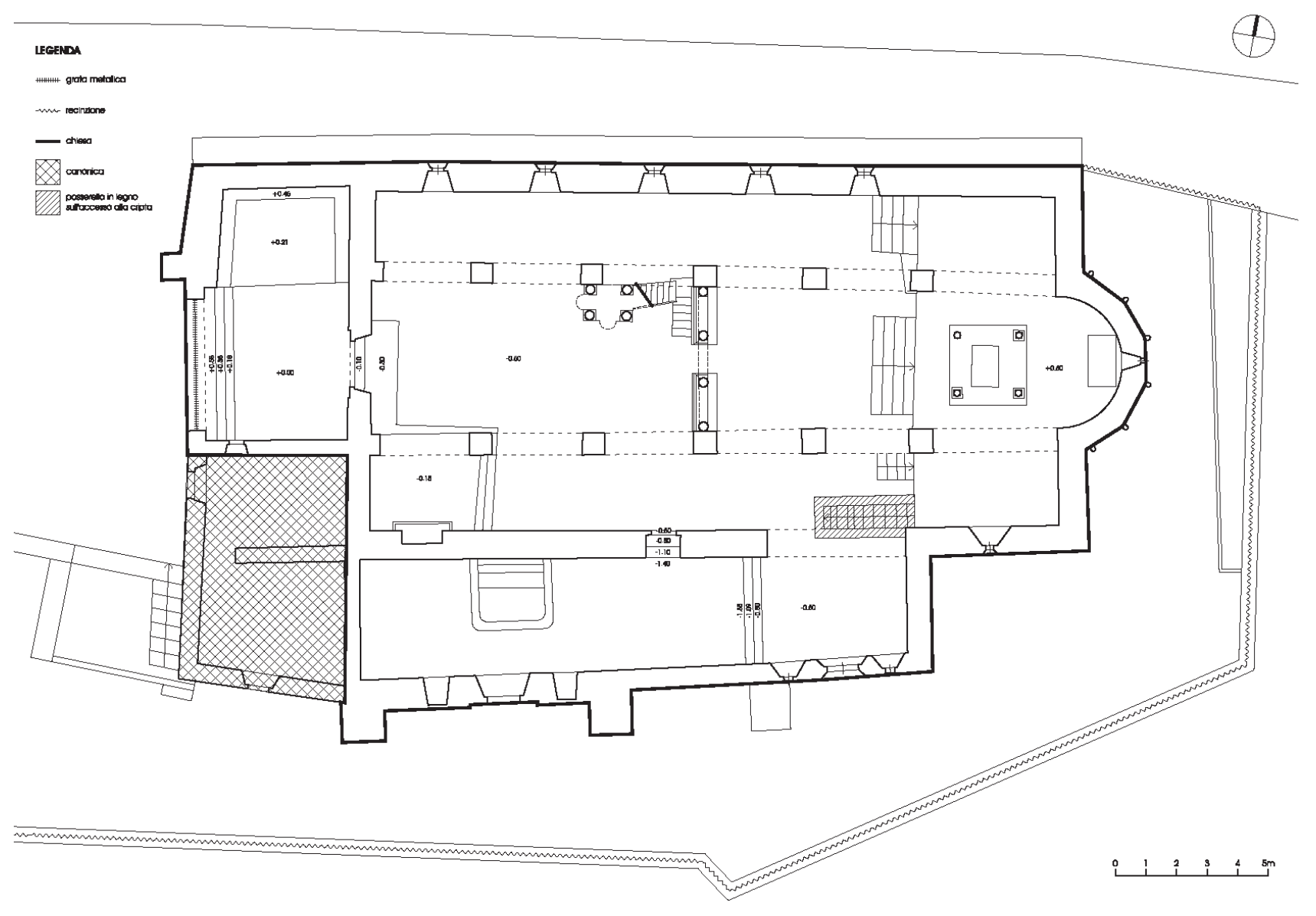

Fig 6. La pianta della chiesa di Santa Maria in Valle Porclaneta

Ciò che si evince da quanto appena esposto, al di là dell'esiguità delle fonti documentarie e da una conseguente contraddittoria interpretazione delle stesse, è che, complessivamente, l'abbazia di Santa Maria in Valle Porclaneta è stata sempre oggetto di grande interesse. La stessa letteratura ha subito, nei confronti del complesso in esame, una netta evoluzione, passando, nell'arco di poco più di un secolo, dalla descrizione dei soli temi decorativi e formali all'analisi di problematiche via via più varie e complesse, analizzate scientificamente. Meno approfondito risulta invece uno studio comparativo, in grado di facilitare la comprensione dell'intero complesso architettonico, compreso lo scomparso monastero e, totalmente assenti risultano essere invece le indagini archeologiche.

Liter che si è cercato di seguire per giungere ad individuare le fasi di vita del cenobio, tra le quali si sono comprese anche le distruzioni e le modifiche sostanziali della fabbrica, è stato quello di sovrapporre i dati dedotti dalla ricerca d'archivio con quelli relativi all'osservazione del dato costruttivo, note le modalità operative del cantiere storico. Si è inoltre tenuto presente che, sulla stratificazione edilizia incidono inoltre, in maniera piuttosto consi- stente, anche i fenomeni di degrado, che vanno parimenti interpretati e incrociati agli altri. Per la chiesa di S. Maria in Valle Porclaneta (fig. 6), dunque, le principali fasi riconosciute sono, ad oggi, sette, come evidenziato di seguito (figure 7 e 8 ).

\section{$1^{a}$ fase - Preesistenza al 1077}

A causa della completa distruzione del monastero, non è possibile stabilire l'esatta consistenza del complesso prima di questa fase. Gli unici dati a disposizione, derivati dagli studi epigrafici, dall'osservazione degli allineamenti di due lati dell'aula, le cui murature corrispondenti hanno uguale spessore, dallo studio comparativo effettuato con fabbriche tipologicamente, spazialmente e cronologicamente affini e dall'analisi dell'apparato figurativo interno alla chiesa, consentono di affermare che in questa fase il complesso abbaziale fosse già funzionale, dotato di una chiesa, un chiostro, gli ambienti necessari allo svolgimento della vita monastica e quelli destinati al lavoro giornaliero. Data la sua posizione rispetto al nartece e alcune valutazioni di carattere geometrico e formale, il campanile, addossato all'aula, potrebbe essere già stato realizzato. 


\section{LEGENDA}

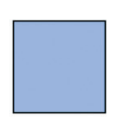

VII fase

(XIX sec.-sisma e restauri)

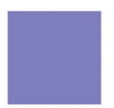

$V$ fase

(XM-inizi XIX sec.- abbandono e degrado)

\section{$\checkmark$ fase}

(prima metà $X V$ sec.-lavori sul fronte sud)

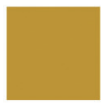

$N$ fase

(fine Xll sec.-distruzione convento?)

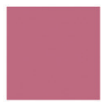

III fase

(seconda metà XIII sec,-realizzazione abside)

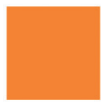

II fose

(seconda metà Xll sec. - ambone e ciborio)

I fose

(preesistenza - Xl sec.)

struthure potizzote

Fig. 7. Legenda relativa alla suddivisione in fasi del complesso

II ${ }^{a}$ fase - Aggiunta degli arredi liturgici nel 1150 (seconda metà del XII secolo)

L'interno della chiesa viene completato con l'aggiunta del ciborio e dell'ambone, realizzati dalla bottega ambulante dei maestri Roberto e Nicodemo come testimoniato dall'epigrafe che attesta la data dell'intervento. Nel 1160 circa si verifica nell'area una scossa sismica, riportata dal Chronicon di Fossae Novae, in seguito alla quale viene probabilmente rimossa la schola cantorum originaria, sostituita dal recinto presbiteriale per realizzare il quale, nel lato destro, vengono utilizzati elementi di reimpiego estranei al sito.

\section{III ${ }^{a}$ fase - Seconda metà del XIII secolo}

Viene realizzato l'abside poligonale romanico ad ordini di colonne sovrapposti e paramento in conci di pietra squadrati. All'interno dell'aula, probabilmente nell'ambito dello stesso cantiere, si realizzano le quattro colonne a sostegno della trabeazione lignea dell'iconostasi e, con tutta probabilità, la trabeazione stessa.
IV ${ }^{a}$ fase - Fine del XIII secolo

Al 1268, anno della battaglia dei Campi Palentini, si fa storicamente risalire la distruzione del monastero di cui, forse, riesce a salvarsi solo l'ambiente longitudinale addossato alla chiesa, sino a questo momento utilizzato probabilmente da filtro tra il monastero e la chiesa e, da adesso in poi, come navata aggiunta.

\section{$V^{a}$ fase - Prima metà del xV secolo}

A questa fase appartengono i lavori sul fronte meridionale della chiesa, nel quale si provvede ad inserire la bifora affiancata dalle due monofore, probabilmente reimpiegate, e a realizzare un nuovo accesso, ottenuto tamponando quello precedente, sul quale viene murato il bassorilievo della Vergine. I lavori di generale rinnovamento dell'edificio sono promossi dai conti di Celano, come attestato dallo stemma inserito accanto alla bifora sul fronte sud. In questa fase di fermento si provvede inoltre a realizzare, all'interno dell'aula, la maggior parte degli affreschi e, probabilmente, a modificare il portale di ingresso.

\section{$V I^{a}$ fase - XVI secolo-prima metà del XIX secolo}

Data la completa assenza di dati documentari relativi a questo periodo e la parallela mancanza di tracce costruttive significative, si deduce che, probabilmente, in questo lasso di tempo si sia verificato il completo abbandono del complesso sul quale inizia ad accentuarsi il processo di degrado.

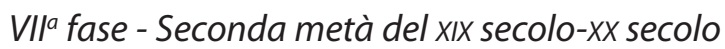

Si susseguono incessantemente gli episodi di restauro della chiesa, tra i quali, con effetti più significativi, quelli relativi alla rimozione del campanile in facciata (1932), alla demolizione e al successivo rifacimento del fronte settentrionale (con asportazione del terreno addossato e successiva creazione della strada carrabile, 1932/35), al consolidamento strutturale di gran parte dell'edificio e degli arredi liturgici (1942/47), al rifacimento interno ed esterno dell'intero piano pavimentale (1967), alla sistemazione dell'area esterna con rimozione del terreno circostante (1967) e, infine, all'adeguamento antisismico dell'intera struttura (1990).

La suddivisione in fasi appena illustrata, che deriva pertanto da un approccio per così dire 'indiretto' nei confronti della fabbrica, emerge, indipendentemente dai problemi legati all'esiguità delle fonti documentarie, alla loro disorganicità e, spesso, all'incongruenza della letteratura presa in esame, dalla difficoltà interpretativa creata dalla spinta antropizzazione del sito e dal succedersi di eventi naturali, un interrogativo lecito, ossia se il manufat- 


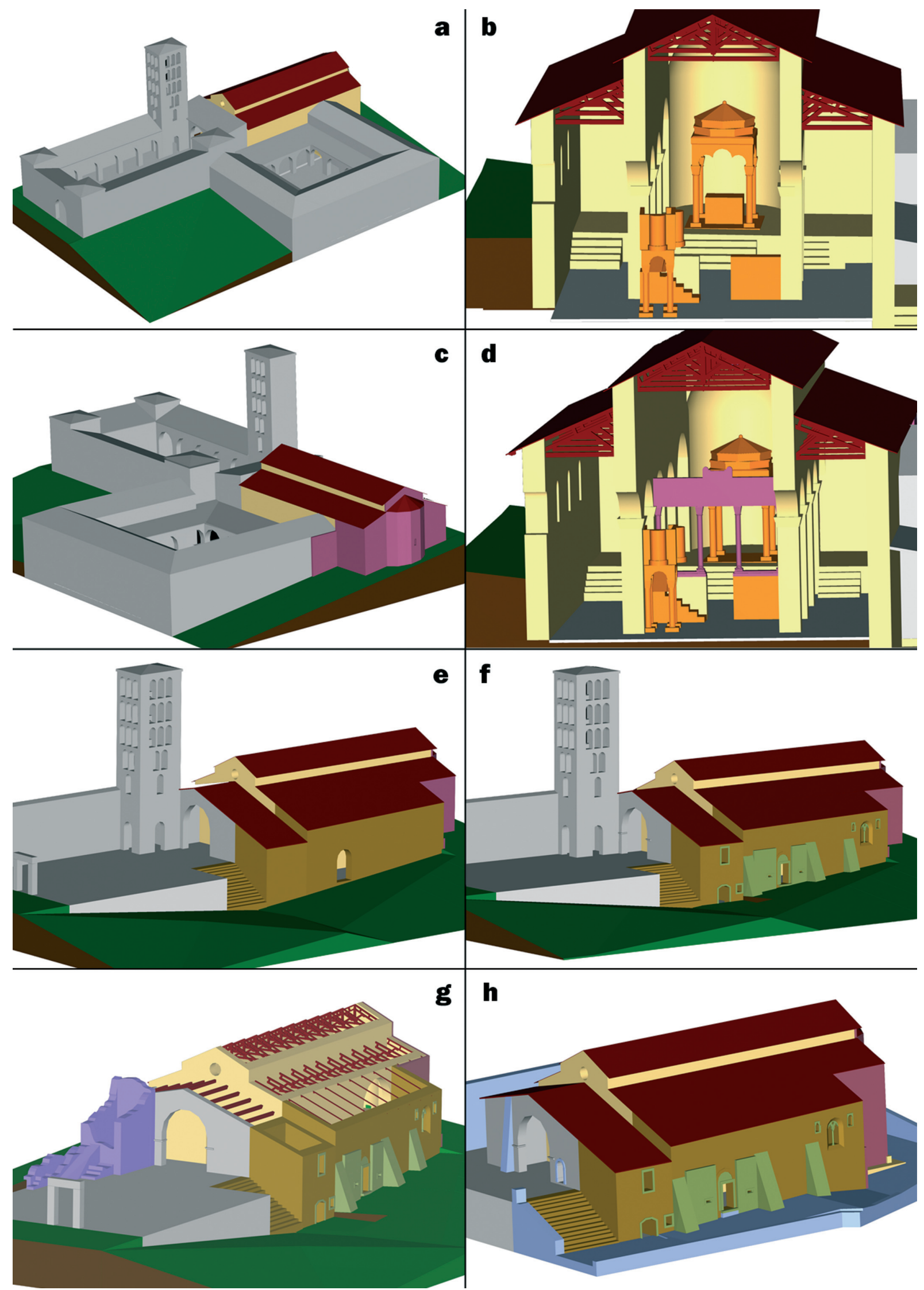

Fig. 8. a) Ricostruzione virtuale, da sud-ovest, del monastero nella la fase individuata; b) ricostruzione relativa alla lla fase; c), d) ricostruzione virtuale della Illa fase; e) ricostruzione virtuale della IVa fase; f) ricostruzione virtuale della Va fase; g) ricostruzione virtuale della Vla fase; $\mathrm{h}$ ) ricostruzione virtuale della VIla fase 


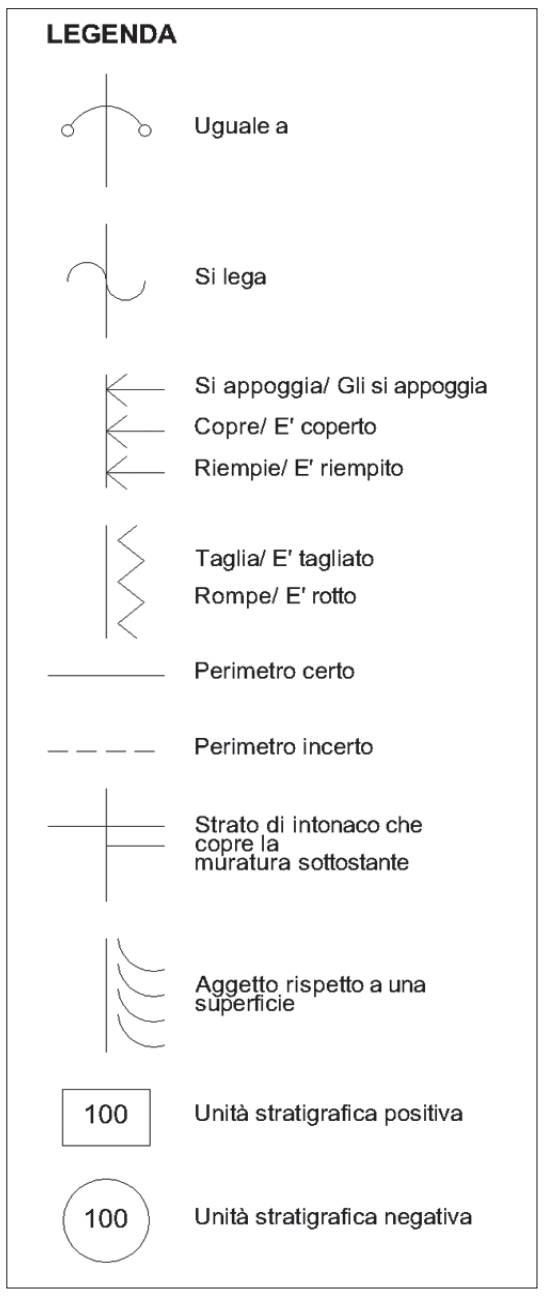

Fig. 9. Legenda dei simboli utilizzati per l'analisi stratigrafica

to in questione possa ancora considerarsi un'opera medievale, di chiaro stampo benedettino o meno.

Infatti, agli occhi degli addetti ai lavori, utilizzando un'espressione medica, l'accanimento terapeutico verificatosi nei confronti dell'edificio, in particolar modo nell'ultimo secolo, con il susseguirsi continuo degli interventi di restauro, ha dato luogo ad un organismo che, probabilmente, si può considerare analogo solo dal punto di vista formale a quello, originario, dell'XI secolo.

A riprova dell'affermazione precedente, infatti, tra le fasi riconosciute, risultano preponderanti, la prima e l'ultima, che possono essere considerate, per tempi e risultati, due grandi fasi costruttive a tutti gli effetti: la prima che ha prodotto il manufatto originale e la seconda che, trasformando profondamente il primo, ci ha consegnato un prodotto moderno e, per così dire 'funzionale'.

Nel paragrafo seguente, vedremo come solo il confronto diretto tra il dato materiale e il 'processo' che lo ha portato a divenire ciò che oggi appare, è possibile effettuare valutazioni oggettive sulla consistenza, natura e autenticità del manufatto. A tale scopo dunque, quanto analizzato sinora

in maniera indiretta, viene ora indagato tramite gli strumenti propri dell'archeologia, come l'analisi stratigrafica, in questa sede interpretata, e sintetizzata, all'interno di un GIS 'verticale'.

\section{ANALISI STRATIGRAFICA INFORMATIZZATA: IL GIS DEI FRONTI DELLA CHIESA DI S.MARIA IN VALLE PORCLANETA}

Chiarito pertanto il motivo che ha portato a testare il GIS 'verticale' su alcune parti della chiesa di Santa Maria in Valle Porclaneta, unito alla ferma convinzione dell'evidente vantaggio rappresentato dall'uso di una piattaforma informatica unica, progettata per raccogliere, gestire ed analizzare una molteplicità di dati differenti tra loro, in particolare laddove risulta evidente la mancanza di omogeneità tra i dati a disposizione, si è proceduto all'analisi stratigrafica di due dei fronti dell'edificio ecclesiastico ${ }^{58}$.

L'analisi stratigrafica sugli elevati della chiesa di S. Maria in Valle Porclaneta ha interessato esclusivamente i fronti esterni est e sud, ritenuti i più significativi soprattutto in quanto ritenuti gli unici testimoni ${ }^{99}$ di importanti tracce materiali dell'evoluzione architettonica della fabbrica (in particolare a sud) e dei restauri avvicendatisi (in particolare ad est). La metodologia seguita si basa in entrambi i casi sulla classica realizzazione dei due fotopiani utilizzati come base per il rilievo stratigrafico ${ }^{60}$. A questi ha fatto seguito, sul campo, il riconoscimento delle differenti USM, selezionate individuando sulle superfici murarie le tracce delle discontinuità presenti, e i caratteri peculiari di ogni elemento riconoscibile. Tali dati sono stati registrati, in sito, su un supporto trasparente sovrapposto a ciascun fotopiano, sul quale sono state in seguito riportate, con apposita simbologia (figura 9), le relazioni tra le parti, e poi informatizzati, alla fine di ogni campagna, in ambiente CAD. Tale procedura, apparentemente semplice, si è nella pratica dimostrata più complessa del previsto, a causa, in numerosi punti, della perdita significativa di sequenze

\footnotetext{
58 Tutto lo studio in oggetto è relativo alla tesi di dottorato: Trizio, I., 2007, Sperimentazione di tecniche di rilevamento archeologico in edifici benedettini della Marsica. Problemi di metodo, applicazioni e risultati: il caso-studio di Santa Maria in Valle Porclaneta, tesi di dottorato in Archeologia Medievale, Università degli Studi dell'Aquila (supervisori proff. D. Fiorani, F. Redi).

${ }^{59}$ Il fronte nord infatti, come emerso nel paragrafo precedente è stato completamente ricostruito nel secolo scorso; quello ovest, invece, è interamente ricoperto da uno spesso strato di intonaco.

${ }^{60}$ I due fotopiani sono stati messi a punto elaborando, con il software di fotogrammetria monoscopica MSR 3.0 della Rollei, numerose riprese fotografiche, ottenute con una fotocamera digitale Fujifilm finePix S5200. Le immagini, modificate a partire dai dati metrici ricavati dai rilievi della Soprintendenza dell'Aquila, sono poi state importate, e quindi ricomposte, all'interno di un software CAD (AutoCAD 2004 dell'Autodesk).
} 


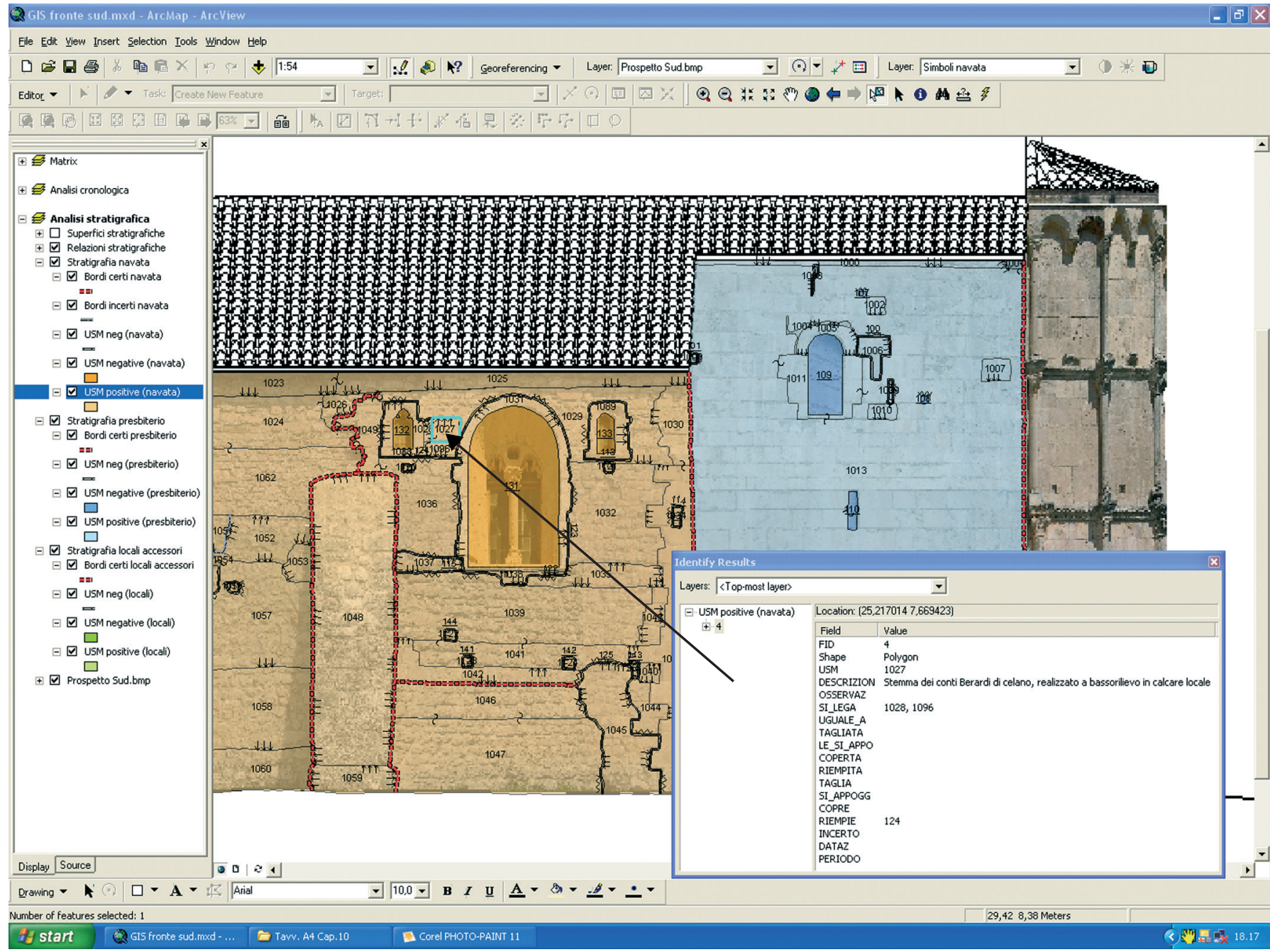

Fig. 10. Schermata del software ArcMap8.3 con il progetto GIS del fronte sud. Si noti come, i dati relativi a ciascuna USM si possano visualizzare, all'interno di una maschera, tramite semplice selezione dell'USM stessa

stratigrafiche, ascrivibili soprattutto ai recenti restauri ${ }^{61}$. L'analisi, particolarmente compromessa dal punto di vista stratigrafico, è stata però conclusa ugualmente, ricorrendo, ogni qualvolta si è ritenuto necessario e possibile, al supporto delle informazioni archivistiche disponibili.

Il numero piuttosto consistente di dati raccolti, peraltro di natura diversa, ha messo in evidenza l'esigenza di associare, su un identico supporto digitale, il rilievo grafico, il rilievo stratigrafico, le immagini e qualsiasi altra informazione relativa ad ogni singola USM.

La strada percorsa è stata quindi quella di progettare un singolo GIS per ogni fronte in esame.

Ciascun GIS, realizzato con il software ArcMap8.3 della ESRI, è stato concepito in modo da raggruppare, all'interno di uno stesso data frame, i vari layers contenenti le unità stratigrafiche e le relazioni reciproche opportuna-

${ }^{61}$ In particolare quello effettuato dalla Soprintendenza dell'Aquila negli anni novanta del secolo scorso. mente importate dalla loro precedente elaborazione in $\mathrm{CAD}$, con una simbologia modificata per esigenze legate alle caratteristiche grafiche del software, mentre, in data frame distinti, si sono tenute separate l'analisi stratigrafica e la cronologia risultante.

Come anticipato in precedenza, l'analisi stratigrafica è stata condotta sul supporto informatico come normalmente avviene su supporto cartaceo: il vero vantaggio derivante dall'utilizzo di questo metodo sta nello sfruttare le relazioni topologiche derivanti dalla vettorializzazione (e quindi georeferenziazione) di ciascuna unità stratigrafica. E' stato infatti possibile agganciare, a ciascuna USM, il database con la descrizione della stessa, la relativa documentazione fotografica e le relazioni con quelle contigue.

Attraverso una semplice interrogazione, per ciascuna USM è possibile visualizzare, all'interno di una finestra o come riga della tabella del database, tutte le informazioni in genere contenute all'interno delle schede ministeriali, 


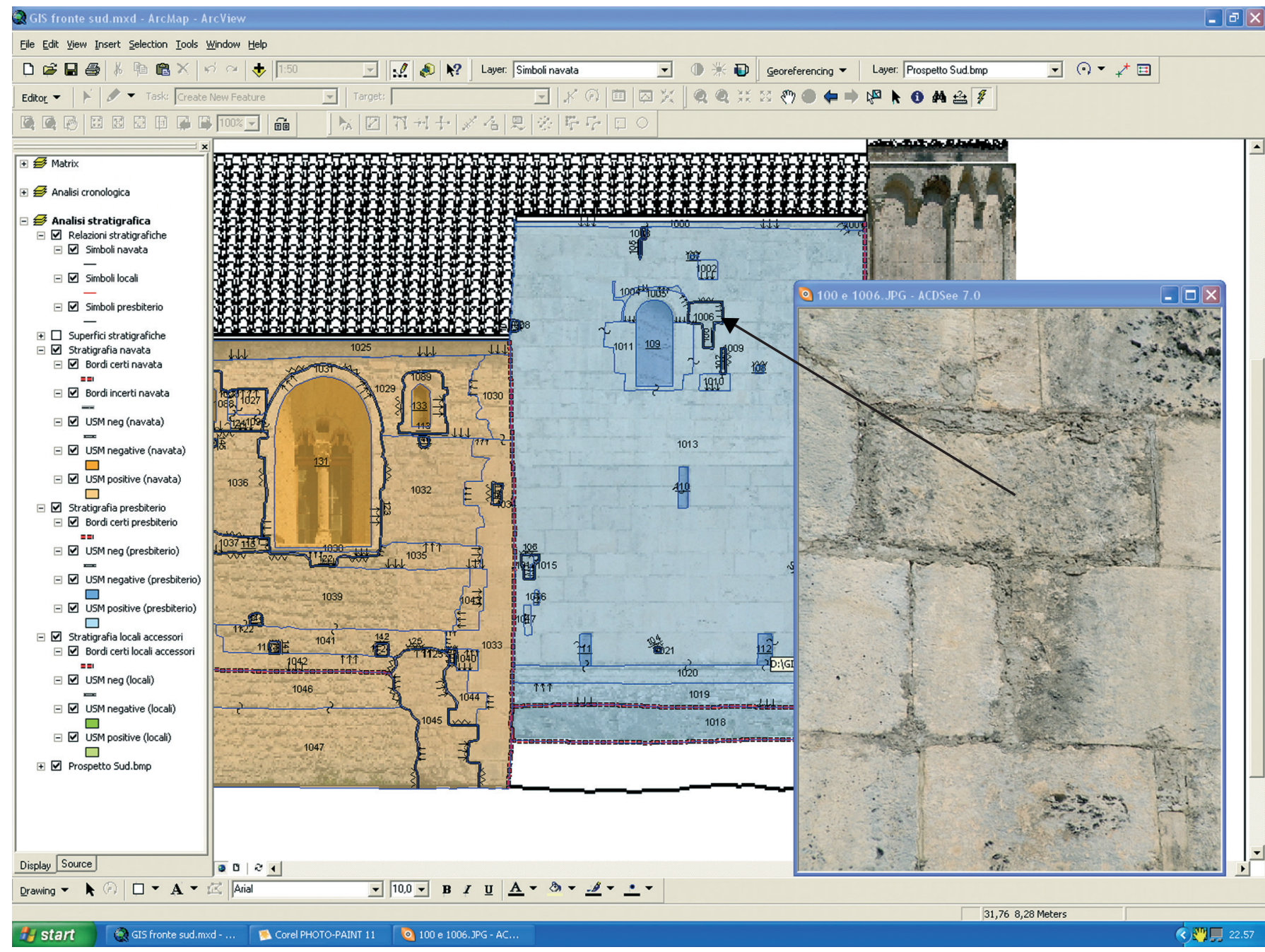

Fig. 11. Schermata del software ArcMap8.3 con il progetto GIS del fronte sud. Si noti come, oltre ai dati contenuti nel database, a ciascuna USM sia stato possibile associare la relativa immagine, visibile all'interno di una finestra, attivabile con una semplice selezione dell'USM stessa

con il vantaggio di poter contemporaneamente osservare l'USM nella sua posizione originale, all'interno del suo contesto architettonico (figura 10).

Un semplice hyperlink consente di accedere poi, per ciascuna USM interrogata, alla visualizzazione del relativo materiale fotografico contenuto all'interno del database (figura 11).

Sebbene l'approccio metodologico sia stato identico in entrambe i fronti, $i$ risultati ottenuti sono decisamente diversi fra loro. Il fronte sud, infatti, che, stratigraficamente parlando, appariva il più complesso tra i due, ha fornito dei risultati assolutamente in linea con la suddivisione in fasi costruttive ipotizzata nel precedente paragrafo. Al contrario, il fronte est, la cui situazione, almeno a giudicare dall'omogeneità della tecnica costruttiva utilizzata, sembrava piuttosto lineare, ha dato delle risposte assolutamente inaspettate e non soltanto dal punto di vista storicocronologico.

\section{Il fronte sud}

Le notevoli dimensioni del fronte in esame, lungo circa 20 $\mathrm{m}$, hanno reso indispensabile, prima di qualsiasi altra operazione, una sua suddivisione in cinque differenti tratti (figura 12), per ognuno dei quali è stata successivamente effettuata la lettura stratigrafica.

Le unità stratigrafiche identificate, per ciascun tratto analizzato, sono state distinte in positive e negative e, quelle negative, a loro volta, rappresentate graficamente attraverso poligoni pieni o semplici contorni (figura 13). La numerazione utilizzata per le unità stratigrafiche negative, distinta graficamente da quella delle positive in quanto caratterizzata dal carattere sottolineato, è partita dal valore 100 , posizionato sulla superficie del presbiterio, per terminare, con il valore 140 , all'interno della superficie degli ambienti accessori alla chiesa. Le USM positive invece, partite dal valore 1000 , posizionato sulla superficie del presbiterio, terminano con il valore 1119 , in corrispondenza degli ambienti ac- 


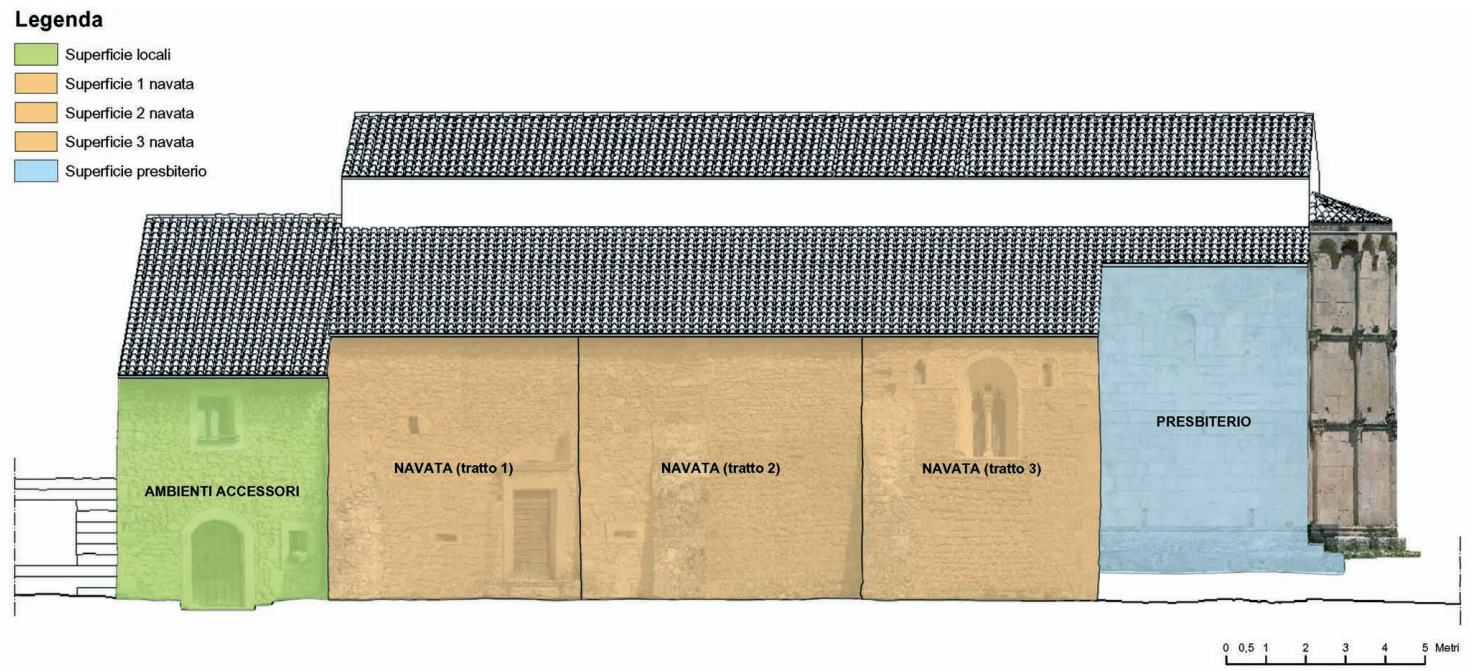

Fig. 12. Schermata del software ArcMap8.3 in cui si evidenzia la suddivisione del fronte sud in tratti. La colorazione che contraddistingue le superfici di ciascun tratto è la stessa utilizzata per la relativa lettura stratigrafica

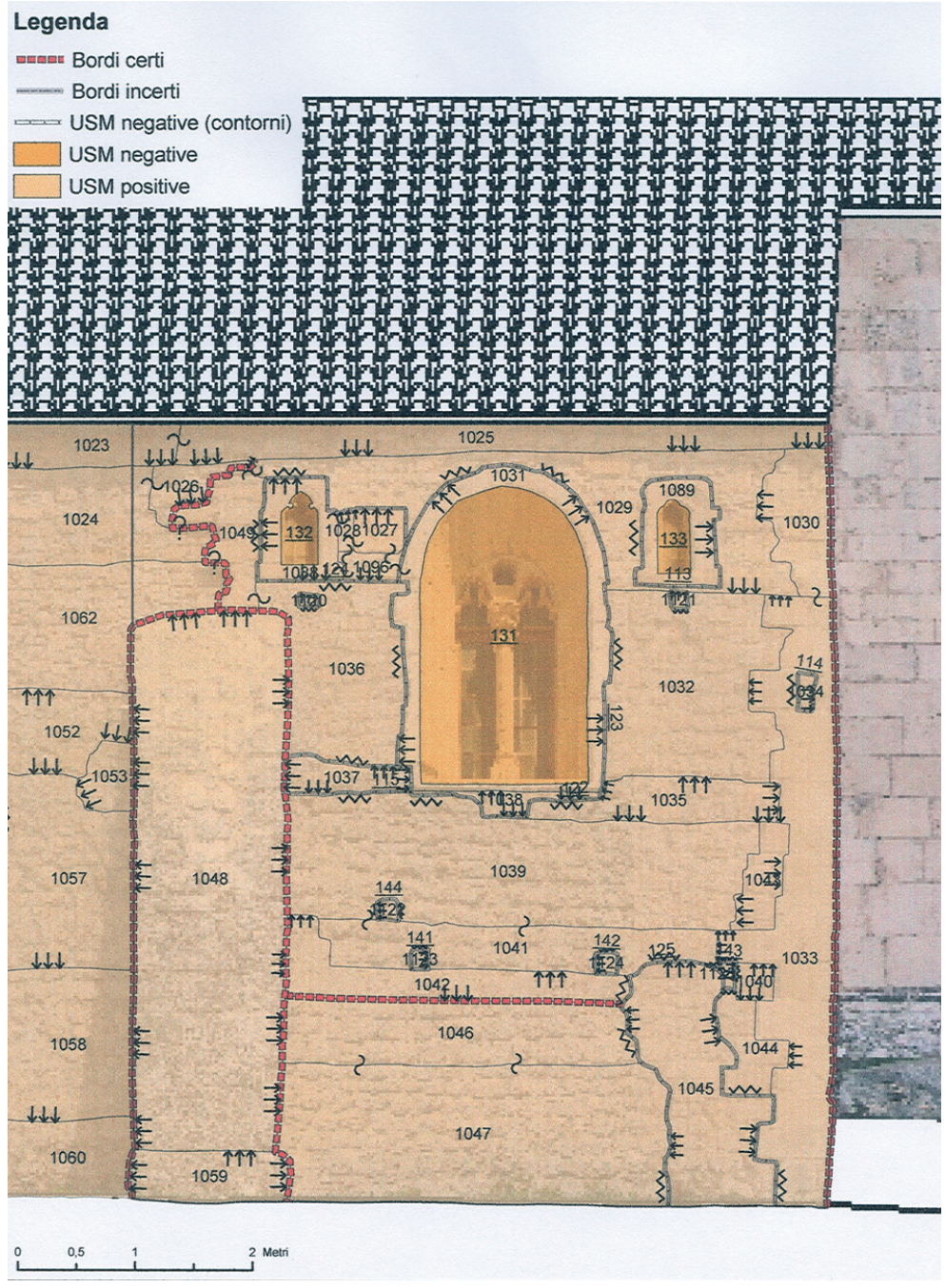

Fig. 13. Schermata del software ArcMap8.3 in cui si visualizza, attraverso una zoomata, il terzo tratto della navata longitudinale aggiunta con le USM individuate e relative relazioni stratigrafiche 


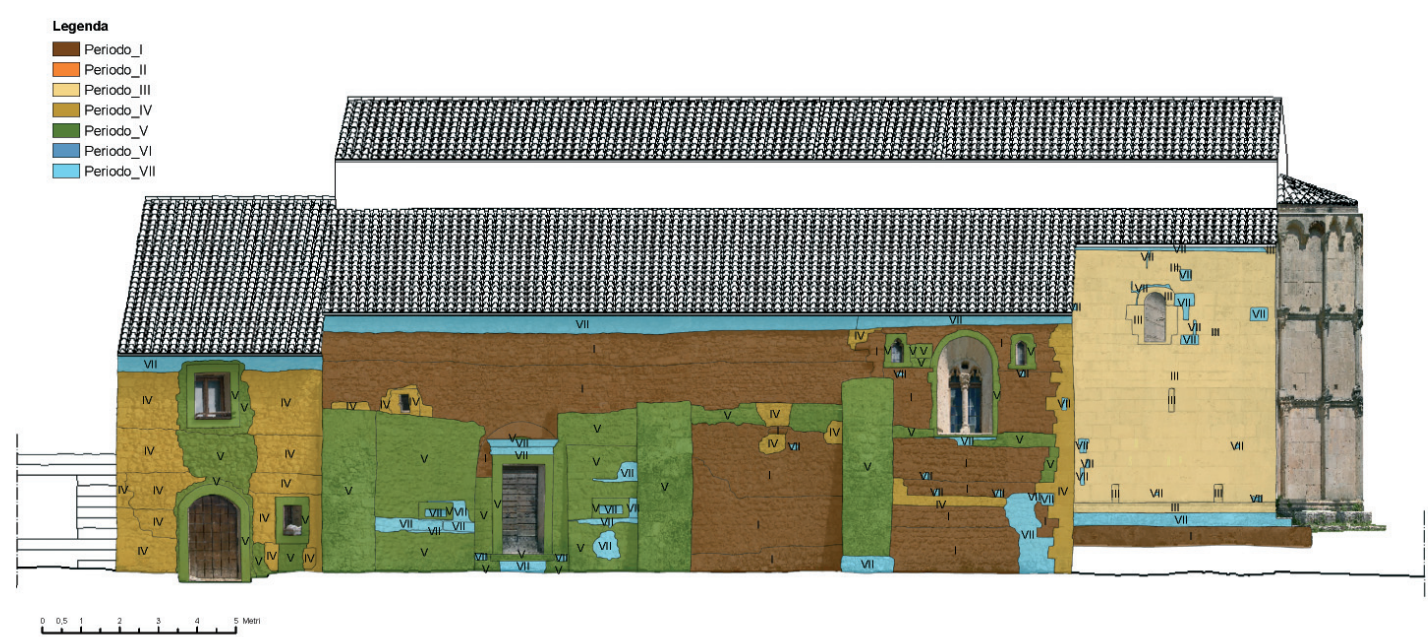

Fig. 14. Schermata del software ArcMap8.3 in cui, passando al dataframe della cronologia, si sovrappone, alle USM del fronte sud la tavola sinottica della ricostruzione cronologica ipotizzata, dedotta dalla datazione delle sottostanti USM

cessori. Sulle varie superfici sono stati inoltre riportati, laddove presenti e chiaramente visibili e riconoscibili, i bordi incerti e i bordi certi che delimitano le superfici analizzate.

Come anticipato in precedenza, l'analisi stratigrafica sul fronte sud ha sostanzialmente riconfermato, e in alcuni casi puntualizzato, i risultati e, quindi, la stessa suddivisione in fasi costruttive, cui si era pervenuti, con indagini 'indirette' nel precedente paragrafo. Tutte le relazioni stratigrafiche tra le parti, anche quando poco intelligibili (come nel caso dei tratti 1 e 3 della navata aggiunta), hanno dato luogo, dopo essere state interpretate e poi collocate all'interno dei relativi matrix, ad una successione cronologica lineare sia dal punto di vista storico che tecnico-costruttivo (figura 14). Poche sono le situazioni veramente dubbie, come ad esempio nel caso dell'USM 1049, in corrispondenza dell' ispessimento della parete a lato della monofora di sinistra nel tratto 3 della navata, in cui non è stato possibile risalire alla relazione con le vicine USM 1024 e 1062, o come nel caso dell'USM 1051 del tratto 2, circondata da un bordo incerto che sembrerebbe, dalla forma, suggerire la presenza di una mensola o la base d'appoggio di un arco poi in seguito tamponato. Proprio quest'ultima osservazione, però, trova una possibile giustificazione nell'ipotesi, che questo fronte, in origine, costituisse una sorta di passaggio tra l'aula ecclesiastica e gli ambienti più privati del cenobio e, quindi, uno dei lati del chiostro, presumibilmente caratterizzato dalla presenza di numerosi archi a tutto sesto.

Escluso dunque i casi isolati appena menzionati e le difficoltà oggettive di ricostruire le sequenze stratigrafiche in una situazione che i numerosi interventi hanno resa incerta e lacunosa, non si rilevano ulteriori osservazioni a proposito di questo fronte per il quale, globalmente, è possibile confermare la suddivisione in fasi costruttive precedentemente esposta e graficamente rappresentata.

\section{II fronte est}

Anche nel caso del fronte est, per condurre l'analisi stratigrafica, si è dovuti ricorrere ad una suddivisione della superficie totale in quattro tratti differenti, così da evitare la gestione contemporanea di un numero eccessivo di USM (figura 15). Tra le quattro superfici risultanti, quella relativa alla navata longitudinale aggiunta è l'unica a non essere completamente rivestita dalla cortina in conci squadrati che caratterizza gli altri tre e a mostrare, di primo acchito, la situazione più articolata. In realtà, dall'analisi stratigrafica è emerso come, anche i tre tratti rimanenti, omogenei dal punto di vista della tecnica costruttiva, presentino caratteristiche di indubbio interesse.

Per quanto riguarda la parete di fondo della navata longitudinale aggiunta, la prima osservazione è relativa alla presenza di una malta grossolana, individuata dall'USM 3003 , che si sovrappone ad un largo tratto di muratura, ricoprendo anche le adiacenti USM (la 3002 e 3004). Questo dato, considerando anche le caratteristiche delI'USM 3016, relativa alla porzione di paramento superstite, che presenta un andamento dei corsi decisamente inclinato e atipico per tale tipo di muratura, ci porta ad ipotizzare come la configurazione attuale derivi dal rifacimento di gran parte della superficie in oggetto, probabilmente dovuto ad un crollo, in conseguenza di qualche sisma, o al riadattamento di questa porzione del monastero, dopo la distruzione del 1268. Inoltre, questo primo tratto analizzato è caratterizzato anche dalla presenza di tre tagli circolari (identificati attraverso le USM 301, 302 e 303), relativi ai fori, praticati prima dell'intervento di 
consolidamento degli anni novanta, per effettuare i sondaggi orizzontali nella muratura.

Per quanto riguarda invece il tratto a sinistra dell'abside poligonale, corrispondente alla parete di fondo della navata destra, su un'area piuttosto ampia, si è riscontrata la presenza dell'USM 3043, costituita da un intonaco di colore scuro, sottile, che copre a tratti la superficie lapidea sottostante, concentrandosi nella parte di muratura in alto a destra. La presenza dei lacerti di intonaco che rivestono il paramento in conci squadrati, originariamente concepito per essere lasciato a vista, non è molto chiara. Probabilmente, però, è da collegarsi allo stesso intervento cui si accennava in precedenza, di rifacimento della parete fondale della navata aggiunta, nell'ambito del quale si è pensato di intervenire, con un rivestimento, anche su questo tratto. Una caratteristica comune ad ognuno dei tre tratti in conci squadrati è relativa alla presenza di forti disconnessioni fra gli elementi del paramento, le più significative consistenti nelle USM 312, 313, 314 per il tratto a sinistra dell'abside, e nelle 319,320 , $321,322,323$ per il tratto di paramento che costituisce l'abside, (figura 16) e le 335, 339 e 340 per quanto concerne il tratto a destra dell'abside. Tutte queste unità stratigrafiche negative, probabilmente derivanti da un'azione sismica parallela alla superficie muraria, sono state a loro volta riempite con vari tipi di malta, dalle caratteristiche differenti. Dall'analisi stratigrafica, infatti, è emerso che le malte utilizzate per riempire i giunti, sono principalmente di cinque tipi: una di colore bianco, con una granulometria degli inerti piuttosto grossolana e ben adesa al supporto, presente solo nell'ultimo dei tre ordini dell'abside (USM 3075); due di colore grigio scuro, differenti non solo per il tipo di posa in opera ma anche per la granulometria degli inerti utilizzati, prevalenti nell'ordine centrale dell'abside (USM 3051 e 3052) e, infine, due di colore grigio chiaro, distinguibili l'una dall'altra per gli inerti di differente granulometria e, soprattutto per il tipo di posa in opera. Tra queste ultime, quella con inerti più fini, caratterizzata da un'accuratissima stilatura dei giunti (USM 3089) sembra la più recente tra quelle analizzate, ed è concentrata nella fascia inferiore di tutti e tre i tratti; quella caratterizzata invece da inerti più grossi (USM 3085 e 3041) appare, tra tutte, la più antica $e$, in genere, si riscontra tra i giunti di dimensioni maggiori (intor-

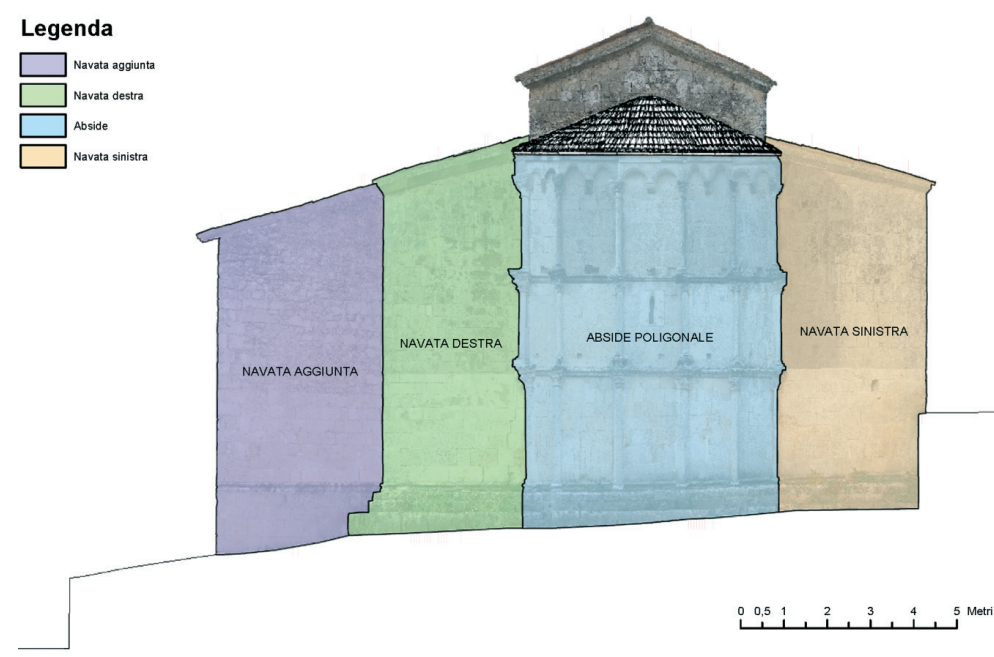

Fig. 15. Schermata del software ArcMap8.3 in cui si evidenzia la suddivisione del fronte est in tratti. La colorazione che contraddistingue le superfici di ciascun tratto è la stessa utilizzata per la relativa lettura stratigrafica

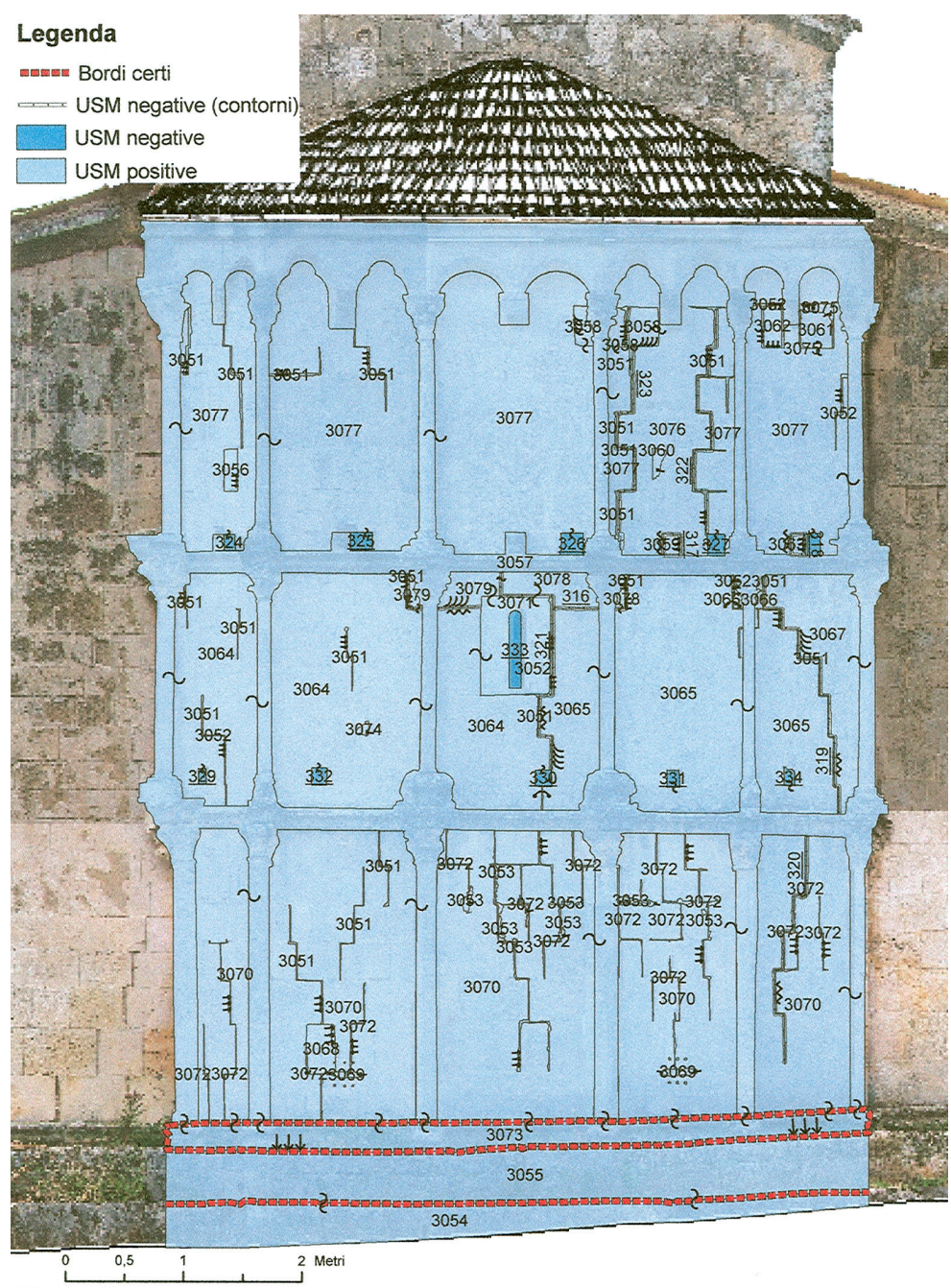

Fig. 16. Schermata del software ArcMap8.3 in cui si visualizza, attraverso una zoomata del fronte est, il tratto relativo all'abside poligonale con le USM individuate e relative relazioni stratigrafiche 
no ai centimetri). Sulla fascia inferiore dell'abside, inoltre, è stata riscontrata la presenza diffusa di tracce di stucco bianco (USM 3053), databili, dalla sequenza stratigrafica, all'ultimo intervento effettuato sul paramento, nel 1990 e, inoltre, sempre in questo stesso tratto sono visibili i segni lasciati dalla prova effettuata nel 1988 con i martinetti piatti (USM 3069), riscontrabile in due diversi punti. Infine, resta da osservare che la tavola sinottica relativa alla cronologia del fronte est (figura 17), esattamente come nel caso del fronte sud, rispetta la suddivisione in fasi costruttive indicata in precedenza, con fase costruttiva prevalente relativa al rivestimento in conci squadrati; mentre, il dato più interessante emerso dall'analisi stratigrafica è legato invece alla mappatura e al riconoscimento delle differenti malte utilizzate tra i giunti, operazione che ha consentito di riconoscere, sul manufatto, e ordinare cronologicamente, le stilature dei giunti effettuate nei diversi interventi di restauro.

\section{CONCLUSIONI}

Il duplice intento perseguito nell'ambito di questa ricerca, quello cioè della 'conoscenza' di un manufatto storico attraverso lo studio integrato delle fonti scritte a quelle materiali, unitamente alla sperimentazione di un ausilio tecnologico in grado di facilitare tale compito, ha consentito di riordinare le particolari vicende costruttive e manutentive dell'edificio in esame e di scioglierne alcuni importanti nodi.

Ciò che è emerso chiaramente nell'iter seguito, a partire dall'analisi del contesto storico e territoriale, passando per la lettura critica della letteratura e dei documenti esistenti, sino ad arrivare alla ricostruzione degli interventi di restauro, è che i due distinti approcci, quello teorico e quello pratico, non hanno valore se non insieme: ne è riprova il fatto che solo facendo convergere tutte le informazioni all'interno del GIS, strumento che ha consentito l'analisi stratigrafica informatizzata, è stato confermato quanto definito, soltanto in linea ipotetica, nella prima parte.

Sebbene la graficizzazione e l'immissione di tutti i dati e di tutte le informazioni riguardanti le varie parti che compongono le porzioni della fabbrica in esame sia una procedura lunga e per certi versi ripetitiva, la certezza che il progetto GIS ottenuto sia, in ogni momento, implementabile ed aggiornabile e consenta sempre una rapida interrogazione delle USM raccolte o la possibilità di effettuare analisi statistiche relative, ad esempio, alla datazione delle stesse, è un vantaggio da mettere in evidenza e che, probabilmente, da solo merita di approfondire tale strumento operativo.
Altra considerazione da evidenziare riguarda, infine, come la combinazione di saperi, approcci e lessici completamente diversi tra loro, appartenenti ora all'archeologia e alla storia dell'architettura, ora all'informatica e all'ingegneria, possa risultare sicuramente faticosa, soprattutto se affidata ad uno stesso individuo, ma dimostra che, probabilmente, è anche l'unica percorribile quando si desidera realizzare la conoscenza 'globale' di un bene ai fini della sua conservazione.

\section{Bibliografia}

Angeloni, V., 2000, Santa Maria in Valle Porclaneta (Sec.XI). Storia, Arte, Leggende e Tradizioni, Avezzano (L'Aquila), Sogeas.

Baracchini, C.; Lanari, P.; Ponticelli, P.; Parenti, R.; Vecchi, A., 2005, SICaR: un sistema per la documentazione georeferenziata in rete, in G. Biscontin; G. Driussi (a cura di), Sulle pitture murali: riflessioni, conoscenze, interventi. Atti del convegno di studi, Bressanone 12-15 luglio 2005, Marghera-Venezia, Arcadia ricerche, pp. 735-747.

Bartolomucci, C., 2004a, Una proposta di 'cartella clinica' per la conservazione programmata, in "Arkos. Scienza e restauro dell'architettura", n. 5, pp. 59-65.

Bartolomucci, C., 2004b, Santa Maria di Collemaggio. Interpretazione critica e problemi di conservazione, Roma, Palombi.

Biallo, G., 2002, Introduzione ai sistemi informativi geografici, Roma, MondoGIS.

Chronicon Monasterium Casinensis, Lib.III, cap. LXI, in Hoffmann, H. (a cura di), 1980, Die Chronik von Montecassino, MGH, Scriptores, XXXIV, Hannoverae, Ed.Hoffmann.

Bindi, V., 1889, Monumenti storici artistici degli Abruzzi. Studi di V.B. con prefazione di F. Gregorovius, Napoli, Giannini \& F., vol. II.

Chierici, U., 1945, Relazione sull'attività dell'Ufficio nel quadriennio 1942-45, Aquila.

D’Antonio, M., 2003, Abbazie benedettine in Abruzzo, Pescara, Carsa.

Castenetto, S. - Galadini, F. (a cura di), 1999, 13 gennaio 1915. Il terremoto nella Marsica, Roma.

De Nino, A., 1904, Sommario dei monumenti e degli oggetti d'arte descritti da Antonio De Nino, Vasto (Chieti), Anelli.

De Spirito, G., 2002, Su talune iscrizioni medievali di S.Maria in Valle Porclaneta, in Luongo, 2002, pp. 436-458.

Diploma di Lotario III del 1137, in Fabiani, L., La terra di S.Benedetto. Studio storico-giuridico sull'Abbazia di Montecassino dall'VIII al XIII secolo, Miscellanea Cassinese 33-34-42, Badia di Montecassino, 1968-80, vol. II, 427.

Doglioni, F., 1997, Stratigrafia e restauro. Tra conoscenza e conservazione dell'architettura, Lint, Trieste.

Dragonetti, G., 1765, Difesa del Regio Padronato di S.Maria della Valle Porcanete, Napoli.

Ferrando Cabona, I. 1998, Problemi di datazione in Archeologia dell'Architettura, in "Archeologia dell'Architettura», n. III, pp. 75-79.

Forte, M., 2002, I Sistemi Informativi Geografici in archeologia, Roma, MondoGIS.

Gabrielli, G. (a cura di), 2001, Usi e consumi dell'informazione geografica: atti della terza conferenza di MondoGIS. Roma, 23-25 maggio 2001, Roma, Ed. MondoGIS.

Gaiani, M., 2002, Dal numerico al visuale: strategie di rappresentazione renderingbased, in Biagini, C. (a cura di), Information technology ed automazione del progetto, Firenze, University Press, pp. 21-57.

In Volo sull'Isola d'Elba: un'applicazione della tecnologia 3D Interactive Map, in «MondoGIS», settembre/ottobre 2003, n. 38, pp. 49-52.

Grossi, G., 2004, Marsica sacra. Chiese, Celle e Monasteri (IV-XII secolo), Avezzano (L'Aquila).

Gavini, I. C., 1927, Storia dell'architettura in Abruzzo, Milano-Roma, Bestetti e Tumminelli, s.a. [ma 1927], vol. I, pp. 50-53, 71-72, 176-187, 292, 346-353, 367, n. 25, 465-466, vol. II, pp. 136, 337. 
Harris, E.C., 2003, The stratigraphy of standing structures, con alcune considerazioni in nota di R. Parenti, in "Archeologia dell'architettura", VIII, pp. 9-14.

Liguori, M. C.; Pescarin, S.; Diamanti, T.; Guidazzoli, A.; Mauri, M.A.; Felicori, M., 2003, Dal GIS alla Realtà Virtuale. Applicazioni per i Beni Culturali e il Decision Making, in "MondoGIS», n. 37, pp. 17-20.

Luongo, G. (a cura di), 2002, La terra dei Marsi. Cristianesimo, cultura, istituzioni. Atti del convegno di Avezzano 24-26 settembre 1998, Roma, Viella.

Mancini, R., 2003, Viaggiare negli Abruzzi, vol. I, L'Aquila, Textus, pp. 293-305.

Mancuso, A. - Tognon, G., 2003, Un sistema informativo per la salvaguardia del patrimonio artistico e architettonico di Venezia, in «MondoGIS», n. 37, pp. 3134.

Mannoni, T., 1994, Venticinque anni di archeologia globale - 3. Caratteri costruttivi dell'edilizia storica, Genova.

Mastroddi, M., 1999, I monumenti danneggiati nella Marsica, in Castenetto Galadini 1999, pp. 373-403.

Mingotto, L., 1999, La cripta della basilica patriarcale di Aquileia. Disegno e rilevamento archeologico dell'architettura storica, in "Archeologia dell'Architettura", n. IV, pp. 159-180.

Moretti, M., 1972, Restauri d'Abruzzo (1966-1972), Roma, De Luca, pp. 254257.

Nardecchia, P., 2004, Note d'arte abruzzese tra la Marsica e il Carseolano, Carsoli (L’Aquila), Ass. Culturale Lumen.

Niccolucci, F., 2001, Utilizzo di strumenti free sotware per GIS nel campo dei beni culturali: un esempio di applicazione al patrimonio archeologico, in Gabrielli, pp. 201-208.

Parenti, R., 1988, Le tecniche di documentazione per una lettura stratigrafica dell'elevato, in Archeologia e restauro dei monumenti, pp. 305-333.

Parenti, R., 1992, Fonti materiali e lettura stratigrafica di un centro urbano: $i$ risultati di una sperimentazione "non tradizionale», in "Archeologia Medievale», XIX, pp. 7-62.

Phoebonius, M. 1678, Historiae Marsorum, vol.III, Napoli, testo latino e traduzione a cura dell'I.N.E.S.A., Roma, De Cristofaro, 1991.

Pierotti, P. - Quirós Castillo, J.A., 2000, Archeologia dell'architettura e storia dell'architettura: due discipline a confronto, in G.P. Brogiolo (a cura di), II Congresso nazionale di archeologia medievale (Brescia, 2000), Firenze, All'Insegna del Giglio, pp. 377-380.

Ricci, C., 1915, in AA.VV., I danni all'arte nei paesi battuti dal terremoto del 13 gennaio 1915, Roma, Calzone.
Salonia, P. - Negri, A., 2001, ARKIS-NET: un WebGIS per la diffusione della conoscenza sul patrimonio costruito storico, in Spagnoletti, pp. 599-603.

Salonia, P. - Negri, A., 2002, Conservazione del patrimonio costruito storico: un sistema per l'integrazione e la gestione di dati eterogenei, in Gabrielli, pp. 189-194.

Salvatori, F., 2002, Geografia e cartografia: da un'idea del mondo alla sua gestione, in Spagnoletti, pp. 17-19.

Santa Maria in Valle Porclaneta di Rosciolo, 2002, a cura della Soprintendenza per i Beni Architettonici e per il Pesaggio, per il Patrimonio Storico, Artistico e Demoetnoantropologico dell'Abruzzo - L'Aquila, L'Aquila.

Sonnino, E., S.Maria in Valle Porclaneta. Note sulla storia conservativa e le caratteristiche tecnico-stilistiche di alcune opere attraverso il loro restauro, in Luongo, 2002, pp. 427-436.

Spagnoletti, E. (a cura di), 2002, In rete con la comunicazione geografica: atti della quarta conferenza di MondoGis. Roma, 22-24 maggio 2002, Roma, Ed. MondoGIS.

Treccani, G.P., 1996, Archeologia stratigrafica e conservazione del costruito: alcuni obiettivi condivisi, in "Archeologia dell'architettura", I, pp. 139-150.

Trizio, I., 2007, Sperimentazione di tecniche di rilevamento archeologico in edifici benedettini della Marsica. Problemi di metodo, applicazioni e risultati: il casostudio di Santa Maria in Valle Porclaneta, tesi di dottorato in Archeologia Medievale, Università degli Studi dell'Aquila (supervisori proff. D. Fiorani, F. Redi).

Trizio, I., 2008, La chiesa di S.Maria in Valle Porclaneta, in Fiorani, D. (a cura di), 2008, Finiture murarie e architetture nel medioevo, Roma, Gangemi, pp. 103-126.

\section{Fonti archivistiche consultate}

- Archivio Centrale dello Stato di Roma, Ministero della Pubblica Istruzione, fondo Antichità e Belle Arti, II versamento (scavi e antichità - musei - gallerie e pinacoteche - monumenti divisi per province), II serie, 1891-1897: busta 14 (monumenti dell'Aquila e provincia).

- Archivio Storico della Soprintendenza ai Beni Ambientali, Architettonici, Artistici e Storici dell'Aquila, cartella E.E.M. 907.

- Archivio Diocesano dei Marsi, fondo C/78/71528.

Recibido: 23 de septiembre de 2009 Aceptado: 10 de noviembre de 2009 